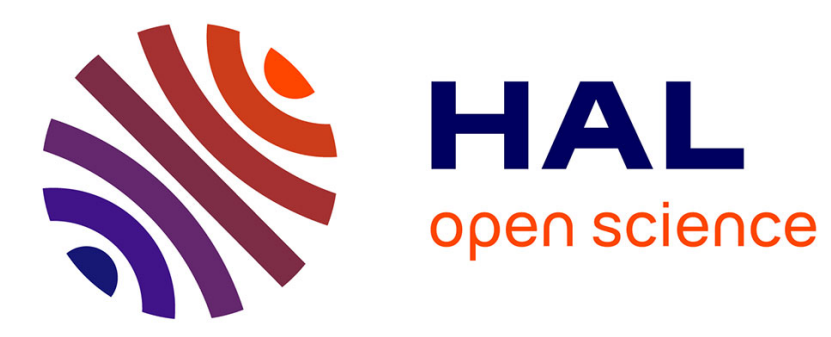

\title{
Short fiber composite reinforcements
}

Julien Férec, Patrice Laure, Luisa Silva, Michel Vincent

\section{To cite this version:}

Julien Férec, Patrice Laure, Luisa Silva, Michel Vincent. Short fiber composite reinforcements. Composite Reinforcements for Optimum Performance, Elsevier, pp.627-669, 2021, 10.1016/B978-0-12819005-0.00020-4 . hal-03096046

\section{HAL Id: hal-03096046 \\ https: / hal-mines-paristech.archives-ouvertes.fr/hal-03096046}

Submitted on 4 Jan 2021

HAL is a multi-disciplinary open access archive for the deposit and dissemination of scientific research documents, whether they are published or not. The documents may come from teaching and research institutions in France or abroad, or from public or private research centers.
L'archive ouverte pluridisciplinaire HAL, est destinée au dépôt et à la diffusion de documents scientifiques de niveau recherche, publiés ou non, émanant des établissements d'enseignement et de recherche français ou étrangers, des laboratoires publics ou privés. 


\begin{abstract}
The chapter focuses on fibre reinforced thermoplastics or polymer composites which are mainly produced by injection and compression molding using particles having mostly cylindrical shape (short or long fibers made up of glass, carbon or flax). The aim of this fibre addition is to improve the mechanical properties of final parts. However these properties depends on the fibre orientation and therefore the knowledge of link between processing condition and final fiber orientations has a major importance.

First the chapter contains results of experimental observations on fibre length distribution, concentration and orientation for complex enough situations in order to describe problems encountered in real industrial processes. It is explained how to get experimental measurements on orientation and interaction tensors from image analysis on polished cross sections or in situ micro-tomography. These information are useful to validate models presented in the next sections.

The models describing the evolution of fibre orientation in a flow motion are mainly based on macroscopic tensors and Folgar Tucker's equation. We present also extensions of this equation which takes into account of non-Newtonian behaviour of polymer, confinement effect and interaction between fibers. Finally, a variety of theories predicting the total stress of fiber suspensions in a Newtonian and complex fluid are exposed.

In the next section, an industrial software and its inherent numerical methods are described. Examples of numerical computations are presented for typical situations. Then the influence of the coupling between the fiber orientation and rheological behaviour of the suspension are analysed. The comparison with experimental data concerning fibre orientation prediction gives information on the validity and the influence of various parameters associated to these models.
\end{abstract}

Key words: Thermoplastic Composite, Reinforced polymers, Fibre orientation, Finite Element Computation

Chapter title: Short fibre composite reinforcements

\title{
Author details
}

Julien Férec

Institut de Recherche Dupuy de Lôme (IRDL)

UMR CNRS 6027

Université Bretagne Sud

56100 Lorient

France 


\section{Patrice Laure}

Laboratoire J.-A. Dieudonné

CNRS UMR 6621

Université de Nice-Sophia Antipolis

Parc Valrose, 06108 Nice Cedex 02

France

Luisa Silva

High Performance Computing Institute

Ecole Centrale de Nantes

1 Rue de la Noë

44300 Nantes

France

Michel Vincent

Cemef

UMR CNRS 7635

Mines ParisTech

BP 207

06904 Sophia Antipolis Cedex

France

e-mail:

julien.ferec@univ-ubs.fr

patrice.laure@unice.fr

luisa.rocha-da-silva@ec-nantes.fr

michel.vincent@mines-paristech.fr 


\section{Section headings}

20.1 Introduction

20.2 Observations

20.2.1 Fibre length distribution

20.2.2 Fibre concentration

20.2.3 Fibre orientation

20.2.4 Skin/core structure

20.3 Models

20.3.1 Fibre orientation: Jeffery model

20.3.1.1 A single fibre in a Newtonian Fluid

20.3.1.2 Effects of non-Newtonian fluids

20.3.1.3 Confinement effects

20.3.1.4 Influence of fibre interactions

20.3.2 Fibre orientation: Folgar Tucker model

20.3.2.1 Closure approximation

20.3.2.2 Interaction coefficient: theoretical, numerical and experimental determination

20.3.3 Fibre orientation: recent models with anisotropic fibre interaction

20.3.4 Fibre orientation: non-Newtonian fluids and confinement effects

20.3.5 Rheological models

20.3.5.1 Generic form with rheological coefficients $N_{p}$ and $N_{S}$

20.3.5.2 Slender body approximation and ellipsoidal particle theories

20.3.5.3 Models with interaction tensor for concentrated suspension

20.3.5.4 Models for non-Newtonian matrix suspension

20.4 Computation of fibre orientation in injection moulding

20.4.1 Numerical methods

20.4.2 Representation of orientation

20.4.3 Rectangular plaque with inlet gate

20.4.4 U-shaped part with thin walls and cross ribs

20.4.5 Square plaque with ribs

20.5 Conclusions 


\subsection{Introduction}

The chapter focuses on fibre reinforced thermoplastics. There are two types of materials. Short fibre reinforced pellets are obtained by mixing glass fibres and a thermoplastic matrix, polypropylene or polyamide for instance. The fibre content is usually between 30 and $50 \mathrm{wt} \%$ and the fibre length is generally distributed around a mean value of $500 \mu \mathrm{m}$. Long fibre reinforced pellets are obtained by continuous impregnation of a fibre, by a pultrusion-like process. After solidification, the impregnated fibres are cut at a length around $10 \mathrm{~mm}$. The fibre length is the same as the pellet length. Both materials are semi-product, adapted for fast production processes such as injection moulding or extrusion.

As any composite, their properties are function of the performances of the matrix, fibres and interface between matrix and fibres, but also of the fibre microstructure. This notion covers first the fibre orientation. Indeed, fibres get oriented by the flow, and their properties are anisotropic. It also covers the fibre length: it depends of the initial fibre length in the pellet, but also on the plasticising and injection steps of the process. Lastly, there is the fibre concentration: it is usually around 30-50 wt\% that is about half in volume for glass fibres, according to the glass and polymer densities. But the transport of the fibres by the polymer may be heterogeneous and fibre-rich or fibre poor region may be found.

The second section of this chapter reviews the experimental observation, and the variation of fibre length distribution, fibre concentration and orientation are discussed. The experimental procedures giving these data are also briefly described. The third section presents the models describing the evolution of fibre orientation in the flow motion. The most recent models which deal with concentrated suspensions are also discussed, as well as the coupling with the rheological behaviour. Finally, numerical computations are performed on three typical examples and the influence of various parameters encountered in the models is analysed. Then, the limitations of these models for real parts are discussed.

\subsection{Observations}

20.2.1 Fibre length distribution 
As mentioned before, fibre length in the moulding depends on the initial fibre length in the pellet, and on the fibre degradation in the screw-barrel system of the plasticising unit and in the mould cavity.

Most methods for measuring fibre length are destructive (Kamal et al., 1986, Chin et al., 1988, Franzen et al., 1989, Gupta et al., 1989, Denault et al., 1989, Akay et al., 1995, Avérous et al., 1997, Davidson and Clarke, 1999). The composite matrix is dissolved or burnt out. The fibre lengths are measured by image analysis of microscope pictures. The number of fibres is large (nearly 3000 per $\mathrm{mm}^{3}$ for $30 \mathrm{wt} \%$ and fibres $300 \mu \mathrm{m}$ long and $15 \mu \mathrm{m}$ in diameter!) and a careful representative selection must be made. The image analysis technique must be able to take into account a large length distribution.

Glass fibre breakage is more important in the plasticising unit (Gupta et al., 1989) than downstream in the runner and mould cavity. It is a function of fibre concentration (Denault et al., 1989, Akay et al., 1995, Tremblay et al., 2000).

Vincent (2009) measured the fibre length distribution in a plaque mould for two reinforced polypropylenes, containing $30 \mathrm{wt} \%$ of short glass fibre, and $30 \mathrm{wt} \%$ of long glass fibres (figure 20.1). The average length of the long fibres, initially $12 \mathrm{~mm}$, reduces to $0.87 \mathrm{~mm}$. The short fibres length, initially $0.56 \mathrm{~mm}$, reduces to $0.41 \mathrm{~mm}$. Fibre breakage for the long fibres composite is more important.

Figure 20.2 shows a plaque $150 \times 150 \times 3 \mathrm{~mm}^{3}$, with seven ribs $25 \mathrm{~mm}$ apart from each other. The rib thicknesses from the entrance to the tip are 1,3, 3, 2, 2, 3, $3 \mathrm{~mm}$. The rib heights are 9, 12, 9, $12,9,12,9 \mathrm{~mm}$. The material is a $40 \mathrm{wt} \%$ long glass fibre reinforced polypropylene (pellet length: 12 $\mathrm{mm}$ ). Table 20.1 shows the average length at different positions for two mould filling flow rate. There is no huge difference between the rib and other regions in the part. The average length reduces by about $20 \%$ along the flow direction. The sensitivity to the flow rate is negligible.

\subsubsection{Fibre Concentration}

Most authors have found small variations of fibre concentration along the flow direction, around 1 to 2 wt $\%$ for a total amount of fibres of $30 \mathrm{wt} \%$ (Hegler and Mennig, 1985; Kubat and Szalanczi, 1974). In the thickness directions, the fibre concentration is higher in the core than in the skin of the moulding, especially for a high fibre loading of 50 wt\% (Kamal et al., 1986; Akay and Barkley, 1991). 
For the same part shown in Figure 20.3, and with the same material, Figure 20.4 shows the fibre concentration. The concentration is higher in the ribs, except in the first one, than in the plaque, but overall the deviation from the initial concentration of the semi-product, $40 \mathrm{wt} \%$, is not very large.

\subsubsection{Fibre Orientation}

For dilute suspension, fibre kinematics was following using 2D optical approach (Taylor, 1923; Binder, 1939; Trevelyan and Mason, 1951; Petrich et al., 2000; Folgar and Tucker, 1984; Stover and Cohen, 1990; Stover et al., 1992; Iso and Koch et al., 1996; Iso and Cohen et al., 1996; Gunes et al., 2008; Yasuda et al., 2002). These aforementioned observations have shown that fibre orientation depends on the type of flow. In a shear flow, a single fibre rotates, but spends most of the time aligned with the flow direction.

In a highly concentrated suspension, when fibres are interacting, fibres are mostly oriented in the flow direction. In an elongational flow, fibres get oriented in a stable position either parallel to the flow with a positive elongation rate, or perpendicular with a negative elongation rate. Interactions with neighbouring particles may disturb this orientation. In a real cavity filling situation, the flow is a complex combination of shear and elongation. Shear reaches a maximum in the skin, and elongation in the core. Elongation always occurs at the junction between the sprue or runners and the cavity itself, because the cross-section of the flow increases. This is why a skin-core structure is often observed in this region. Downstream, if the flow is shear dominated, fibres in the centre are reoriented in the flow direction, but the core region may appear again in case of increase of cross section.

Figures 20.4 show polished cross sections of long glass fibre mouldings (Megally, 2005). In the surface, fibres appear mainly oriented in the flow direction, whereas in the centre, they are perpendicular to it. They are mostly parallel to the plan of the part. Short fibre composites show the same type of orientation. When the fibres are long enough, more than around $500 \mu \mathrm{m}$, some of them are broken during the screw plastication but the remaining longer fibres can be slightly curved. Otherwise, they appear as straight rods. 
In order to be precise about the orientation, it is necessary to quantify it. The fibre orientation distribution function $\psi(\boldsymbol{p}, t)$ is defined by the probability $\psi(\boldsymbol{p}, t) \mathrm{d} \boldsymbol{p}$ of finding a fibre oriented between $\boldsymbol{p}$ and $\boldsymbol{p}+\mathrm{d} \boldsymbol{p}$, where $\boldsymbol{p}$ is a unit vector aligned with a fibre (Prager, 1957). The second order orientation tensor $\boldsymbol{a}_{2}$ is easier to use in comparing two orientation patterns (Hand, 1961). It is defined as the spatial average of the double tensorial product of $\boldsymbol{p}$, and it is symmetrical and positive definite:

$\boldsymbol{a}_{2}=\int_{\boldsymbol{p}} \boldsymbol{p} \otimes \boldsymbol{p} \psi(\boldsymbol{p}, t) \mathrm{d} \boldsymbol{p}$

The trace of the tensor is equal to 1 . For random orientation in space, the diagonal terms are equal to $1 / 3$, and for random planar orientation, to $1 / 2$. For perfect orientation in direction $i, a_{i i}=1$, and the other diagonal values are zero. A diagonal tensor means that the reference frame axes are the principal axes of the tensor.

In order to obtain the orientation distribution function $\psi$ or the second order orientation tensor components, each fibre orientation in a given volume must be determined. Several techniques can be used (see for instance Clark and Eberhardt (2002) for a review), and a useful technique for fibre orientation characterization in molded parts consists in observing a polished cross-section (as depicted in figures 20.4), either by optical or scanning electronic microscopy (Vincent et al., 2005). These observations give a rough idea of the fibre state, and quantification is very important to compare efficiently the different patterns. Cylindrical fibres making a certain angle with respect to the cutting plane appear as ellipses. The measurement of the semi-axes of the ellipse allows the quasi-3D reconstruction and leads to the vector $\boldsymbol{p}$ components in $3 \mathrm{D}$. The image analysis technique must be accurate enough to separate touching fibres or to measure nearly circular fibre cross sections, and corrections must be applied for long fibres for which the probability of intersecting the edge of the observed field is higher. Another important correction is necessary because the probability of observing fibres perpendicular to the observation plane is higher than when they are parallel to it (Bay and Tucker, 1992).

However, these techniques which work well for semi-dilute suspensions have limitations for concentrated fibre suspensions. In addition, they do not offer directly 3D views of fibres and their kinematics. The X-ray microtomography (Shen, et al., 2004) which becomes actually a very popular 
technique can be used. This last technique allows the $3 \mathrm{D}$ reconstruction of the fibres in a given volume (Faessel et al., 2005; Le et al., 2008; Suuronen et al., 2013). With appropriate image analysis procedures, relevant microstructure descriptors such as the position, orientation of fibres and fibrefibre contacts can be extracted (Latil et al., 2011; Guiraud et al., 2012; Wegner et al., 2012; Viguié et al., 2013). Moreover, recent synchrotron X-ray sources have made possible to acquire 3D images at very fast scanning times, and thus to perform real-time 3D in situ observations during the deformation of imaged samples (Limodin et al., 2009; Laurencin et al., 2017). For these concentrated suspensions another parameter which is the number of contact points per fibre can be measured and linked to an interaction tensor $\boldsymbol{b}_{2}$. In fact, assuming a constant length distribution and a no-interpenetration condition, Toll (Toll, 1993; Toll, 1998) expressed the average number of contact points per fibre for slender bodies as follows:

$\bar{z}=\frac{8}{\pi} \phi \beta f$

with $\beta$ is the aspect ratio of fibre defined in section $3, \phi$ the fibre volume fraction and $f$ the trace of the second-order interaction tensor $\boldsymbol{b}_{2}$ defined as follows (Djalili_Moghaddam and Toll, 2005; Férec et al., 2009, Férec et al., 2014):

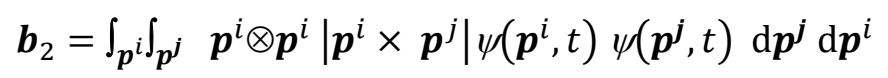

$f=\operatorname{Trace}\left(\boldsymbol{b}_{2}\right)=\int_{\boldsymbol{p}^{i}} \int_{\boldsymbol{p}^{j}}\left|\boldsymbol{p}^{i} \times \boldsymbol{p}^{j}\right| \psi\left(\boldsymbol{p}^{i}, t\right) \psi\left(\boldsymbol{p}^{j}, t\right) \mathrm{d} \boldsymbol{p}^{j} \mathrm{~d} \boldsymbol{p}^{i}$

where the superscript $i$ refers to the test fibre while $j$ is used for the neighboring fibre and $\left|\boldsymbol{p}^{i} \times \boldsymbol{p}^{j}\right|$ is the Onsager potential (Onsager, 1949) which is maximum and equals one when two rods interact orthogonally and is minimum and null when two rods are both parallel.

The figures 20.5 shows the compression of a concentrated suspension of quasi-aligned polymer fiber immersed in olive oil that was studied using in situ observations (Latil et al., 2011). This sample was subjected to a vertical compression and Figure 20.5 shows the evolution of the force measured along the vertical axis and representations of the fibrous structure deducted from X-thomography measurements during the flow. As noted in previous works (Orgéas et al., 2012; Guiraud et al., 2012), Figure 20.5 shows that, quantitatively, the Toll's model forecasts reproduce rather well the experimental evolution of average contacts although the theoretical predictions are slightly higher. 
Another example showing the compression of suspension containing 2770 fibres corresponding to fibre volume ratio of $40 \%$ can be found in (Laurencin, 2017).

\subsubsection{Skin-core structure}

A review of observations in various part geometries, such as plaques or centre gated disks, with different composites can be found in Papathanasiou (1997). The skin-core structure is often observed, the relative thickness of the skin and core layers depending on the processing conditions. At the flow front, fibres are mostly tangential to the front. This is why weld line regions exhibit weaker mechanical properties.

Figure 20.6 shows the gapwise evolution of the orientation tensor component in the flow direction, in a $50 \mathrm{wt} \%$ glass fibre reinforced polyarylamide plaque, for four cavity thicknesses (Vincent et al., 2005, Vincent, 2009). The skin-core structure exists for the two largest thicknesses, but vanishes for the smallest ones. The shape of the plaque entrance, where the core is created, and the high shear rate generated by a small flow gap are responsible for this difference.

\subsection{Models}

20.3.1 Fibre orientation: Jeffery theory

\subsubsection{A single fibre in a Newtonian Fluid}

Jeffery (1922) considered a single rigid ellipsoidal particle with very small dimensions. Thus, the rate of deformation can be considered as homogeneous around the particle, and he supposed that this particle was immersed in a Newtonian fluid. The following time evolution equation of the unit vector $\boldsymbol{p}$ aligned with the particle axis was thus obtained:

$\frac{\mathrm{d} \boldsymbol{p}}{\mathrm{d} t}=\Omega \boldsymbol{p}+\lambda[\dot{\varepsilon} \boldsymbol{p}-(\dot{\varepsilon}: \boldsymbol{p} \otimes \boldsymbol{p}) \boldsymbol{p}]$

where $\dot{\varepsilon}$ is the rate of strain tensor, $\Omega$ is the vorticity tensor, both defined as function of $\boldsymbol{v}$, the velocity field:

$$
\begin{aligned}
& \dot{\varepsilon}(v)=\frac{1}{2}\left(\nabla v+\nabla v^{t}\right) \\
& \Omega(v)=\frac{1}{2}\left(\nabla v-\nabla v^{t}\right)
\end{aligned}
$$


$\lambda$ is a function of the aspect ratio of the particle $\beta=L / D$, with $L$ the length and $D$ the diameter of the fibre. One gets for ellipsoids (both oblate and prolate spheroids):

$\lambda=\frac{\beta^{2}-1}{\beta^{2}+1}$

In this theory, a particle rotates periodically in a simple shear flow, with a period of rotation of:

$T_{f}=\frac{2 \pi}{\overline{\dot{\varepsilon}}}\left(\beta+\frac{1}{\beta}\right)$

where $\overline{\dot{\varepsilon}}=\sqrt{\sum_{i, j} \dot{\varepsilon}_{i j}^{2}}$ is the magnitude of the strain rate tensor. This relation has been experimentally validated (Moses et al, 2001). If the particle is infinitely long (slender body), the particle does not rotate anymore, but tends to orient in the flow direction. In elongation flows, equation (20.4) shows that a particle tends to a stable equilibrium position, parallel or perpendicular to the flow direction when the elongational rate is positive or negative (Tucker and Advani, 1994). The kinetics of orientation are almost independent of the fibre aspect ratio when it is high.

Later, Bretherton (1962) theoretically demonstrated that any rigid body of revolution has a motion in shear flow identical to an ellipsoid, meaning that the theory could be extended to fibre motion provided that the correct value of $\lambda$ is chosen (Petrie, 1999). For straight cylindrical fibre, one gets:

$\lambda=1-\frac{3 K \ln (\beta)}{4 \pi \beta^{2}}$

where $K \approx 5.45$ is a constant determined by Cox (1971) from the experiments of Anczurowski and Mason (1968). Typically, short fibres have a diameter between 10 and $20 \mu m$, and length between 100 and $500 \mu \mathrm{m}$, so that $\beta$ is of the order 5 to 50 , and $\lambda$ is larger than 0.92 .

\subsubsection{Effects of non-Newtonian fluids}

However, few experimental, theoretical, and numerical studies focused on fibre kinematics in shearthinning and viscoelastic fluids, whereas this rheological behavior is typical for industrial polymers, as those that are used in composites. In fact, departures of the fibre orientation from the Jeffery's orbits have been observed for non-Newtonian fluids, mostly for shear flows. Hence, using viscoelastic fluids, fibres were found to align along the shear or the vorticity directions, depending on the Weissenberg number, e.g. by changing the shear rate or the fluid viscoelastic properties (Iso et al., 1996; Gunes et 
al., 2008). These experimental results were also observed numerically and theoretically (Leal, 1975; Brunn, 1977; D'Avino et al., 2014; Borzacchiello et al., 2016). Nevertheless, for power law fluid having a shear thinning behaviour, theoretical considerations and experimental observations showed that the Jeffery's equation is found valid assuming a slender body (Férec et al., 2016; Laurencin et al., 2019).

\subsubsection{Confinement effects}

Moreover, Jeffery-based theories were established for good scale separation conditions, i.e., when the size of fibres is small compared with the typical size of flowing samples. Unfortunately, this critical prerequisite is rarely fulfilled by the composite forming conditions. In many situations, composites flow through narrow channels or in thin mold cavities with gaps being in the same order of magnitude as the fibre length $L$. These flow situations correspond to confined regimes (Schiek and Shaqfeh, 1997; Diamant, 2009; Snook et al., 2012): the scale separation between the sample and its heterogeneities is poor so that fibres are close to the mold walls. It is well-known that when fibres interact with a solid wall, departures from the Jeffery's trajectories and orbits are prone to occur (Moses et al., 2001). Few theoretical studies proposed modifications of Jeffery's equations to account for confinements and/or wall effects (Schiek and Shaqfeh, 1997; Ozolins and Strautins, 2014; Perez et al., 2016, Scheuer et al., 2016; Scheuer et al., 2018, Laurencin et al., 2019). In these models, the particle rotary velocity is split into two terms: the Jeffery rotary component (equation (20.4)) and a confined component which depends on the type of flow and the position of touching walls. In particular, Scheuer et al. (2018) showed that this decomposition holds for non-Newtonian fluids.

\subsubsection{Influence of fibre interactions}

Jeffery's equation (20.4) is valid for dilute suspensions, when the fibre volume fraction $\phi \ll 1 / \beta^{2}$. In the semi-concentrated regime, $1 / \beta^{2} \ll \phi \ll 1 / \beta$, hydrodynamic fibre interactions occur, whereas in the concentrated regime $(\phi \gg 1 / \beta)$, fibre-fibre contacts also appear. Fibre-reinforced thermoplastics are often in this latest regime.

Since in the concentrated regime one cannot follow each fibre, the orientation distribution function, $\psi(\boldsymbol{p}, t)$, defined in the previous section is used. The equation of conservation of $\psi$ is of the Fokker-Planck type and is written (Bird et al., 1987):

$\frac{\partial \psi}{\partial t}+\frac{\partial}{\partial \boldsymbol{p}}\left(\psi \frac{d \boldsymbol{p}}{d t}\right)=0$

For concentrated regimes, Folgar and Tucker (1984) introduced a pseudo-Brownian diffusion, giving 
$\frac{\partial \psi}{\partial t}+\frac{\partial}{\partial \boldsymbol{p}}\left(\psi \frac{d \boldsymbol{p}}{d t}\right)=D_{r} \frac{\partial^{2} \psi}{\partial \boldsymbol{p}^{2}}$

Therefore, the Jeffery equation was modified by introducing this diffusion term which model fibre interactions:

$\frac{\mathrm{d} \boldsymbol{p}}{\mathrm{d} t}=\Omega \boldsymbol{p}+\lambda_{\boldsymbol{p}}[\dot{\varepsilon} \boldsymbol{p}-(\dot{\varepsilon}: \boldsymbol{p} \otimes \boldsymbol{p}) \boldsymbol{p}]-D_{r} \frac{\partial \ln \psi(\boldsymbol{p}, t)}{\partial \boldsymbol{p}}$

This phenomenological term, $D_{r}$, is proportional to the effective deformation rate $\dot{\bar{\epsilon}}$ and to a constant diffusion coefficient $C_{I}$, called the interaction coefficient. However, this useful model fails to produce slow orientation kinetics for rod suspensions as encountered in non-dilute systems due to the interactions (Sepehr et al., 2004). Based on a physical approach at the microscale, Férec et al. (2009) exhibited a pseudo-shape factor, $\lambda_{\boldsymbol{p}}$, that is a function of $\boldsymbol{p}$ :

$\lambda_{\boldsymbol{p}}=1-\phi \widetilde{M} \int_{\boldsymbol{p}^{j}}\left|\boldsymbol{p} \times \boldsymbol{p}^{j}\right| \psi\left(\boldsymbol{p}^{j}, t\right) \mathrm{d} \boldsymbol{p}^{j}$

where $\widetilde{M}$ is a dimensionless scalar that relates the intensity of the friction between particles and the superscript $j$ refers to the neighboring fibre. This term is due to an interaction flux created by the fibrefibre interactions and it is obtained from a global torque produced by neighboring fibres, which acts on the test fibre kinetics. With this is approach, the diffusional terms introduced by Folgar and Tucker (1984) had to be modified and was expressed as

$D_{r}=q \overline{\dot{\varepsilon}} \phi \widetilde{M} \int_{\boldsymbol{p}^{j}}\left|\boldsymbol{p} \times \boldsymbol{p}^{j}\right| \psi\left(\boldsymbol{p}^{j}, t\right) \mathrm{d} \boldsymbol{p}^{j}$

where $q$ is a dimensionless interaction coefficient (similar to $C_{I}$ ).

\subsubsection{Fibre orientation: Folgar Tucker model}

The distribution function is a complete and accurate representation of the orientation state. However, solving the equation (20.10) requires important computational resources, and for industrial applications, it is better and easier to use the second order orientation tensor, $\boldsymbol{a}_{2}$, defined in equation (20.1) . Passing from an orientation distribution function $\psi(\boldsymbol{p}, t)$ to $\boldsymbol{a}_{2}$ implies a loss of information. Thus, one may use higher order tensors, such as $\boldsymbol{a}_{4}$ the fourth-order orientation tensor

$\boldsymbol{a}_{4}=\int \boldsymbol{p} \otimes \boldsymbol{p} \otimes \boldsymbol{p} \otimes \boldsymbol{p} \psi(\boldsymbol{p}, t) \mathrm{d} \boldsymbol{p}$

and may reconstruct $\psi(p, t)$, as suggested by Advani and Tucker (1987) from both tensors. 
To directly solve orientation motion based on the second-order orientation tensor, Lipscomb et al. (1984) provided the following equation, after volume averaging of Jeffery's equation:

$\frac{d \boldsymbol{a}_{2}}{d t}=\Omega \boldsymbol{a}_{2}-\boldsymbol{a}_{2} \Omega+\lambda\left(\dot{\epsilon} \boldsymbol{a}_{2}+\boldsymbol{a}_{2} \dot{\epsilon}-2 \dot{\epsilon}: \boldsymbol{a}_{4}\right)$

For more concentrated solutions, the diffusion term added by Folgar and Tucker (1984) to account globally for fibre interactions gives:

$\frac{d \boldsymbol{a}_{2}}{d t}=\Omega \boldsymbol{a}_{2}-\boldsymbol{a}_{2} \Omega+\lambda\left(\dot{\epsilon} \boldsymbol{a}_{2}+\boldsymbol{a}_{2} \dot{\epsilon}-2 \dot{\epsilon}: \boldsymbol{a}_{4}\right)+2 C_{I} \overline{\dot{\varepsilon}}\left(\boldsymbol{\delta}-3 \boldsymbol{a}_{2}\right)$

This model has been extensively used, and is the standard model. Questions arise when treating this equation: how to compute $\boldsymbol{a}_{4}$ from $\boldsymbol{a}_{2}$ ? What are the admissible values for $C_{I}$ ? How to extend this model to concentrated suspension?

\subsubsection{Closure approximations}

The fourth order tensor $\boldsymbol{a}_{4}$ appears in the time evolution equation for $\boldsymbol{a}_{2}$, thus a closure approximation is needed to approximate $\boldsymbol{a}_{4}$ as a function of $\boldsymbol{a}_{2}$ (since to compute $\boldsymbol{a}_{4}$, one needs also $\boldsymbol{a}_{6}$ ). Tests carried out in simple flows (shear, elongation, or simple combination of both) showed that the closure approximation has a large influence on the quality of the result. The different approaches were summarized in figure 4.3 in (Férec and Aussias, 2015) and we recall here the simplest or more commonly used closure approximations. The simplest approximation is the quadratic closure which gives exact results when fibres are perfectly aligned (Doi and Edwards, 1986):

$a_{4}^{Q}=a_{2} a_{2}$

and the linear closure which is exact for random orientation (Hand, 1962):

$\boldsymbol{a}_{4}^{L}=-\frac{1}{35}\left(\boldsymbol{\delta} \boldsymbol{\delta}+\mathbf{I}+\mathbf{I}^{\dagger}\right)+\frac{1}{7} \boldsymbol{a}_{2} \cdot\left(\boldsymbol{\delta} \boldsymbol{\delta}+\mathbf{I}+\mathbf{I}^{\dagger}\right)+\frac{1}{7}\left(\boldsymbol{\delta} \boldsymbol{\delta}+\mathbf{I}+\mathbf{I}^{\dagger}\right) \cdot \boldsymbol{a}_{2}$

where the three fourth-order isotropic tensors are defined in terms of their Cartesian components as follows (Bird and Curtiss et al., 1987):

$(\boldsymbol{\delta} \boldsymbol{\delta})_{i j k l}=\delta_{i j} \delta_{k l} \quad ; \quad \mathbf{I}_{i j k l}=\delta_{i l} \delta_{j k} \quad ; \quad \mathbf{I}_{i j k l}^{\dagger}=\delta_{i k} \delta_{j l}$

The hybrid closure approximation is a linear combination, depending on the orientation, of the two previous ones (Advani and Tucker, 1990): 
$\boldsymbol{a}_{4}^{H}=\left(1-f_{\boldsymbol{a}_{2}}\right) \boldsymbol{a}_{4}^{L}+f_{\boldsymbol{a}_{2}} \boldsymbol{a}_{4}^{Q}$

where $f_{\boldsymbol{a}_{2}}=1-27 \operatorname{det}\left(\boldsymbol{a}_{2}\right)$ is a scalar measure of orientation in a 3D orientation field, which varies from 0 in the case of a random orientation to 1 in the case of a perfectly aligned orientation. As a result, the hybrid closure is exact when the fibres are in random state or in perfect alignment. However, this closure tends to accelerate the orientation transients in transient shearing flows. Performance studies of theses closure approximations in both shear and elongational flows were analysed in Verleye (1995). It was showed that linear closures may provide non-physical oscillations, which did not happen with quadratic or hybrid closures. The transient state at shorter time is generally overestimated whereas the stationary one reached for larger time is underestimated; fibre alignment prediction is very unidirectional. In elongation flows, linear closure may also give unrealistic values, whereas quadratic or hybrid approximations provide better results, even if they overestimate the transient region.

One other class of closure approximations includes fitted parameters in simple flows, like the orthotropic (Cintra and Tucker, 1995; Wetzel and Tucker, 1999) or the natural closures (Dupret and Verleye, 1999). For orthotropic closures, three selected independent components of $\boldsymbol{a}_{4}$ were developed as a two order polynomial expansion of the two largest eigenvalues of $\boldsymbol{a}_{2}$. For natural closures, the fourth-order tensor was written as a linear function of the invariants of the second-order orientation tensor. Coefficients of the different function corresponding to each approximation were obtained by fitting to the analytical solution of the steady-state orientation distribution function for several flow situations: shear, elongation and combination of both.

Orthotropic closures always provide the best results and are the most commonly used. However for low $C_{I}$ values, the first orthotropic closures proposed by Cintra and Tucker (1995) suffered from non-physical oscillations when used for simple shear and radial diverging flows. Chung and Kwon (2001) have proposed an improvement to overcome this problem. This was achieved by introducing fitted flow data that covers the entire range of fibre orientation states, in particular the combined shear/planar-elongation flow resembling to radial diverging flow. Finally they showed that a third order polynomial expansion of the two largest eigenvalues of $\boldsymbol{a}_{2}$ performed better especially for 
non-homogeneous radial diverging flows with low $C_{I}$ values. Chung and Kwon (2002a) have also developed an extension of natural closures which predicts fibre orientation as accurately as methods based on orthotropic closure with lower computational time. Comparisons of these different approaches are given in Chung and Kwon (2002b).

\subsubsection{Interaction coefficient: theoretical, numerical and experimental determination}

For shear flows, the interaction coefficient value is very important in making good predictions of the steady-state solution of the orientation tensor: if $C_{I}$ is high, the orientation becomes isotropic, whereas for a weak $C_{I}$, fibres tend to align in the flow direction. On the other hand, in elongation flows, $C_{I}$ influences the orientation transient solution: if $C_{I}$ is high, fibre orientation attains the steady state more rapidly than for lower values.

In a shear flow, when $C_{I}$ is around $10^{-4}, a_{11}$ is close to 1 , meaning that fibres are very well oriented in the flow direction. When $C_{I}$ increases, $a_{11}$ decreases, and for $C_{I}=1$, orientation becomes nearly isotropic $\left(a_{11}=1 / 3\right)$. The fibre aspect ratio $\beta$ has a negligible influence on the steady-state attained value, even though Hinch and Leal (1973) showed that for very weak diffusion (a very small interaction coefficient) it may become important.

The value of $C_{I}$ can be determined through theoretical, numerical or experimental fittings. In this last case, it can be difficult to find only one $C_{I}$ that fits in the whole flow range. Bay and Tucker (1992) used an empirical approach, based on experimental values for injected disks and plaques at different polymers and fibre concentrations, to propose:

$C_{I}=0.0814 e^{-0.7148 \phi \beta}$

On the theoretical side, Ranganathan and Advani (1991) proposed a relationship between the interaction coefficient and the distance between fibres, $h$ :

$C_{I}=K \frac{L}{h}$

where $K$ is a constant determined experimentally and $L$ the fibre length.

Direct numerical simulation has also been used to estimate $C_{I}$ values. Yamane et al (1994) modelled fibre-fibre interaction for a Newtonian fluid in shear flow, using lubrication forces. The 
authors obtained very low interaction coefficients $\left(10^{-7}<C_{I}<10^{-4}\right)$. Fan et al (1998) developed a numerical approach to consider both fibre-fibre and hydrodynamic interactions leading to an interaction coefficient that has a tensorial form, which will be discussed in the next section. PhanThien et al. (2002) extended the previous approach and proposed the following form for $C_{I}$ :

$C_{I}=0.03\left(1-e^{-0.224 \phi \beta}\right)$

The values obtained seem of a good order of magnitude $\left(10^{-3}<C_{I}<10^{-2}\right)$. Furthermore, one obtains the interesting result that the interaction coefficient increases with the fibre volume fraction and the fibre aspect ratio. This is a point of discussion, since high volume fractions do not often lead to isotropic orientations, which are the result of Folgar and Tucker's model when one considers a high $C_{I}$. The models discussed in the next section will enlighten this fact.

20.3.3 Fibre orientation: recent models with anisotropic fibre interaction

In the previous standard model (0.16), an isotropic rotary diffusion term was added by Folgar and Tucker. The diffusivity is proportional to the magnitude of the rate of deformation (scalar), through the constant $C_{I}$. Experimental data has shown that, for concentrated suspensions, kinetics of orientation is much slower than are predicted by the standard Folgar and Tucker model. To avoid this effect, Wang et al. (2008) and Phelps and Tucker (2009) introduced the Reduced Strain Closure (RSC) model in order to slow down the fibre orientation kinetics. The authors wrote $\boldsymbol{a}_{2}$ as a function of its eigenvalues $\Lambda_{i}$ and eigenvectors $\mathbf{e}_{i}$, and supposed that the eigenvalue kinetics was slowed by a factor of $\kappa$ and that the eigenvector kinetics was unchanged. The tensorial material derivative of $\boldsymbol{a}_{2}$ was then recalculated, resulting in the following variation of the Folgar and Tucker's model:

$$
\begin{aligned}
\frac{\mathrm{d} \boldsymbol{a}_{2}}{\mathrm{~d} t}=\Omega \boldsymbol{a}_{2}- & \boldsymbol{a}_{2} \Omega+\lambda\left(\dot{\epsilon} \boldsymbol{a}_{2}+\boldsymbol{a}_{2} \dot{\epsilon}-2 \dot{\epsilon}:\left[\boldsymbol{a}_{4}+(1-\kappa)\left(\boldsymbol{l}_{4}-\boldsymbol{m}_{4}: \boldsymbol{a}_{4}\right)\right]\right) \\
& +2 \kappa C_{I} \overline{\dot{\varepsilon}}\left(\boldsymbol{\delta}-3 \boldsymbol{a}_{2}\right)
\end{aligned}
$$

The fourth order tensors $\boldsymbol{l}_{4}$ and $\boldsymbol{m}_{4}$ are function of the eigenvalues and eigenvectors of $\boldsymbol{a}_{2}$ :

$\boldsymbol{l}_{4}=\sum_{i=1}^{3} \Lambda_{i}\left(\mathbf{e}_{i} \otimes \mathbf{e}_{i} \otimes \mathbf{e}_{i} \otimes \mathbf{e}_{i}\right)$ 
$\boldsymbol{m}_{4}=\sum_{i=1}^{3}\left(\mathbf{e}_{i} \otimes \mathbf{e}_{i} \otimes \mathbf{e}_{i} \otimes \mathbf{e}_{i}\right)$

and parameter $\kappa$ was determined by fitting experimental data. For short fibre reinforced thermoplastics, it ranges from 0.05 to 0.2 (Wang et al., 2008). To avoid the use of closure approximations, Wang et al. (2008) have also derived a Fokker-Planck equation including this kinetics slow phenomenon. Nevertheless, it remains computationally expensive when compared with the tensorial form.

Using a different approach, Férec et al. (2009), obtained a similar model. The authors have considered that orientation kinetics becomes slower with semi-concentrated suspensions because of hydrodynamic and fibre-fibre interactions. The force generated by fibre interactions was modelled using a linear hydrodynamic friction coefficient proportional to the relative velocity at the contact point and weighted by the probability for contacts to occur. The modified Jeffery's equations were depicted in equations (20.11-20.13). Starting from this approach, the authors obtained the following orientation evolution equation:

$$
\begin{aligned}
\frac{\mathrm{d} \boldsymbol{a}_{2}}{\mathrm{~d} t}= & \Omega \boldsymbol{a}_{2}-\boldsymbol{a}_{2} \Omega+\lambda\left(\dot{\varepsilon} \boldsymbol{a}_{2}+\boldsymbol{a}_{2} \dot{\varepsilon}-2 \dot{\epsilon}: \boldsymbol{a}_{4}\right) \\
& +\phi \widetilde{M}\left(\dot{\varepsilon} \boldsymbol{b}_{2}+\boldsymbol{b}_{2} \dot{\varepsilon}-2 \dot{\varepsilon}: \boldsymbol{b}_{4}\right)+2 f \phi \widetilde{M} q \bar{\varepsilon}\left(\boldsymbol{I}-3 \boldsymbol{a}_{2}\right)
\end{aligned}
$$

where $f$ is the average number of contacts per fibre defined in equation (20.3), $\widetilde{M}$ is a parameter related to drag, and $q$ is a dimensionless interaction coefficient (namely the group of terms $2 f \phi \widetilde{M} q$ is equivalent to coefficient $C_{I}$ of the standard Folgar and Tucker model (equation (20.16)). The second order interaction tensor $\boldsymbol{b}_{2}$ was already defined in equation (20.3) and can be approximated (Férec et $a l ., 2009)$ by

$\boldsymbol{b}_{2}=\frac{3 \pi}{8}\left(\boldsymbol{a}_{2}-\boldsymbol{a}_{4}: \boldsymbol{a}_{2}\right)$

whereas the fourth-order interaction tensors $\boldsymbol{b}_{4}$ was also approximated through a quadratic closure approximation:

$\boldsymbol{b}_{4}=\frac{\boldsymbol{b}_{2} \otimes \boldsymbol{b}_{2}}{\operatorname{Trace}\left(\boldsymbol{b}_{2}\right)}$ 
In addition, Férec et al. (2009) have demonstrated that their model can be rewritten as a RSC model type. An improvement of this approach can also be found in Férec et al. (2014) in which the Brownian terms was not used but the new closure relation for the fourth-order interaction tensor, $\boldsymbol{b}_{4}$ are proposed (it was developed as a polynomial expansion of three eigenvalue of $\boldsymbol{b}_{2}$ ). The second orientation interaction tensor $\boldsymbol{b}_{2}$, was expressed as a function of the orientation tensors thanks to an invariantbased optimal fitting approximation. For all these expansions, the unknown parameters were obtained using a least-square fitting technique with simple and steady solutions computed from the probability distribution function.

Globally, the RSC model behaves well for short fibre reinforced materials. For long fibre thermoplastics, it quantitatively generates higher flow aligned fibres which do not agree with the experimental values. Thus, anisotropic rotary diffusion (ARD) models have been lately developed (Ranganathan and Advani, 1991; Fan et al., 1998; Phan-Thien et al, 2002; Koch, 1995; Phelps and Tucker, 2009). Ranganathan and Advani (1991) proposed a model in which the interaction coefficient is inversely proportional to the average interfibre spacing (see equation (20.22)). Diffusion is isotropic and not really applicable to long fibre reinforced materials. Fan et al. (1998) and Phan-Thien et al. (2002) developed a rotary diffusion anisotropic model by replacing the interaction coefficient $C_{I}$ by a second order tensor $\boldsymbol{C}$, computed by performing direct numerical simulations of a REV of a concentrated suspension undergoing a simple shear flow (Beaume, 2009). $\boldsymbol{C}$ was then determined using the steady state solution, not exploiting dynamic behaviour. Koch (1995) obtained also an expression for the tensor $\boldsymbol{C}$ using a mechanistic approach, by considering the influence of hydrodynamic fibre-fibre interactions on the orientation development in the semi-dilute regime. This tensor is of the form

$\boldsymbol{C}=\frac{n L^{3}}{\dot{\bar{\epsilon}}^{2} \ln ^{2} \beta}\left[b_{1}\left(\dot{\varepsilon}: \boldsymbol{a}_{4}: \dot{\varepsilon}\right) \boldsymbol{\delta}+b_{2}\left(\dot{\varepsilon}: \boldsymbol{a}_{6}: \dot{\varepsilon}\right)\right]$

with $n$ the number of fibres per unit volume, $b_{1}$ and $b_{2}$ are parameters computed by fitting with analytical orientation values in elongation flows, $\boldsymbol{a}_{6}$ is the sixth-order orientation tensor. The author was then led to the following orientation equation: 
$\frac{\mathrm{d} \boldsymbol{a}_{2}}{\mathrm{~d} t}=\Omega \boldsymbol{a}_{2}-\boldsymbol{a}_{2} \Omega+\lambda\left(\dot{\varepsilon} \boldsymbol{a}_{2}+\boldsymbol{a}_{2} \dot{\varepsilon}-2 \dot{\varepsilon}: \boldsymbol{a}_{4}\right)$

$+\overline{\dot{\varepsilon}}\left(2 \boldsymbol{C}-2\right.$ Trace $\left.(\boldsymbol{C}) \boldsymbol{a}_{2}-5\left(\boldsymbol{C} \boldsymbol{a}_{2}+\boldsymbol{a}_{2} \boldsymbol{C}\right)+10 \boldsymbol{a}_{4}: \boldsymbol{C}\right)$

Since the sixth-order tensor needs to be computed, the Koch model is more computationally costly. It does not provide better results than Folgar and Tucker model for long fibre reinforced thermoplastics, and the quality of results is also dependent on the ratio between $b_{1}$ and $b_{2}$ (if $b_{1}$ is much larger than $b_{2}$, diffusion becomes mostly isotropic and the model close to Folgar and Tucker's one).

Recently, Phelps and Tucker (2009) developed a phenomenological anisotropic rotary diffusion model, using also a second-order tensor to describe interaction, by allowing the diffusion term to depend on the orientation state and on the rate of deformation tensor, using the final expression $\boldsymbol{C}=b_{1} \boldsymbol{\delta}+b_{2} \boldsymbol{a}_{2}+b_{3} \boldsymbol{a}_{2}{ }^{2}+\frac{b_{4}}{\overline{\dot{\varepsilon}}} \dot{\epsilon}+\frac{b_{5}}{\overline{\dot{\varepsilon}}^{2}} \dot{\epsilon}^{2}$

The five scalar parameters $b_{1}, \cdots, b_{5}$ are fitted in order to get steady state orientation solutions in simple shear flow and various elongation flows, ensuring stable steady state positive eigenvalues, as well as physical solutions. The complexity of determining valid parameter sets for this model led Tseng and co-workers (Tseng et al., 2013; Tseng et al., 2016; Tseng et al., 2018) to develop the iARD (improved Anisotropic Rotary Diffusion) model which assumes that anisotropy of diffusion is only controlled by the deformation mode:

$\boldsymbol{C}=C_{I}\left(\boldsymbol{\delta}-4 C_{M} \frac{\dot{\varepsilon}^{2}}{\overline{\dot{\varepsilon}}^{2}}\right)$

where the new parameter $C_{M}$ controls anisotropy and this approach is actually used in Moldex3D. Recently, Bakharev et al. (2018) have introduced a model in which the rotary diffusion tensor depends only the local fibre orientation through eigenvalues of $\boldsymbol{a}_{2}$ :

$\boldsymbol{C}=C_{I}\left(\sum_{i=1}^{3} D_{i} \mathbf{e}_{i} \otimes \mathbf{e}_{i}\right)$

where the coefficients $D_{i}$ are associated to eigenvalues $\Lambda_{i}$ sorted in descending order and these default values of $D_{1}=1, D_{2}=0.8$ and $D_{3}=0.15$ are used in Moldflow (Autodesk Help, 2018) and is denoted as MRD model. A similar model called pARD is proposed in Moldex by Tseng et al. (2018). 
Recently, Favaloro and Tucker (2019) have re-cast the general equation (20.31) in a new, interpretive form that readily identifies an effective scalar interaction coefficient $\widehat{C_{1}}$ and a target orientation tensor $\widehat{\boldsymbol{a}}$ :

$$
\frac{\mathrm{d} \boldsymbol{a}_{2}}{\mathrm{~d} t}=\Omega \boldsymbol{a}_{2}-\boldsymbol{a}_{2} \Omega+\left(\widehat{\hat{\epsilon}} \boldsymbol{a}_{2}+\boldsymbol{a}_{2} \hat{\dot{\epsilon}}-2 \hat{\dot{\epsilon}}: \boldsymbol{a}_{4}\right)+6 \widehat{C_{I}} \dot{\bar{\epsilon}}\left(\widehat{\boldsymbol{a}}-3 \boldsymbol{a}_{2}\right)
$$

where $\widehat{\hat{\epsilon}}=\lambda \dot{\epsilon}-15 \widehat{C}_{I} \dot{\bar{\epsilon}} \widehat{\boldsymbol{a}}, \widehat{C}_{I}=\operatorname{Trace}(\boldsymbol{C}) / 3, \widehat{\boldsymbol{a}}=\boldsymbol{C} / \operatorname{Trace}(\boldsymbol{C})$. The authors re-write a lot of anisotropic rotary diffusion model in this form. Then, they got the parameter needed of each model to fit a steady-state orientation in a shear flow. Finally, the comparisons of transient behaviour shown differences and they concluded that the last model (20.35) is the most attractive as the needed parameters can be easily obtained to measured final orientations.

Finally, the Reduced Strain Closure model and Anisotropic Rotary Diffusion model can be collected in an unique model (so-called ARD-RSC model) which is thus written as:

$$
\begin{gathered}
\frac{\mathrm{d} \boldsymbol{a}_{2}}{\mathrm{~d} t}=\Omega \boldsymbol{a}_{2}-\boldsymbol{a}_{2} \Omega+\lambda\left(\dot{\epsilon} \boldsymbol{a}_{2}+\boldsymbol{a}_{2} \dot{\epsilon}-2 \dot{\epsilon}:\left[\boldsymbol{a}_{4}+(1-\kappa)\left(\boldsymbol{l}_{4}-\boldsymbol{m}_{4}: \boldsymbol{a}_{4}\right)\right]\right) \\
+\dot{\bar{\epsilon}}\left\{2\left[\boldsymbol{C}-(1-\kappa) \boldsymbol{m}_{4}: \boldsymbol{C}\right]-2 \kappa \operatorname{Tr}(\boldsymbol{C}) \boldsymbol{a}_{2}-5\left(\boldsymbol{C} \boldsymbol{a}_{2}+\boldsymbol{a}_{2} \boldsymbol{C}\right)\right. \\
\left.+10\left[\boldsymbol{a}_{4}+(1-\kappa)\left(\boldsymbol{l}_{4}-\boldsymbol{m}_{4}: \boldsymbol{a}_{4}\right)\right]: \boldsymbol{C}\right\}
\end{gathered}
$$

This latest model has shown to be predictive for long fibre thermoplastic composites; even though there is a large number of parameters to fit (Tseng et al., 2017) and it can be used in Moldflow software.

\subsubsection{Fibre orientation: confinement effects and non-Newtonian fluids}

In the sections 20.3.1.3 and 20.3.1.3, extensions of Jeffery's model have been proposed to take into account of confinement effect and the non-Newtonian behaviour of fluid. As the Jeffery equation was not modified for power law fluid, the Folgar and Tucker model discussed previously can always apply. For second-order fluid model Brunn (1977) gave a general expression of particle orientation (or Jeffery equation) which was used by Borzacchiello et al. (2016) to derive a complex mascroscopic description which involves moments up to sixth order of the orientation distribution. Therefore, the authors proposed a simplified modelling framework valid for low Weissenberg number that allows one to extend to viscoelastic suspending fluids the standard Folgar and Tucker model. 
For simple shear flows and moderately confined flow conditions, Mezher et al. (2016) have shown that the orthotropic closure within the classical Folgar and Tucker framework captures the orientation evolution. However, in strongly-confined situations, they concluded that two approaches can be followed: either use of a set of microstructure descriptors richer than the single second-order orientation tensor (Perez et al., 2016), or look for better closure relations involving the second-order moment only that could be fitted in confined flow conditions, following the same rationale that was considered for deriving the orthotropic closure approximation (Chung and Kwon, 2001).

\subsubsection{Rheological models}

In the most general case, we consider that the extra stress tensor is the sum of the contribution of the fluid and of the particles (Batchelor, 1971), and that a reinforced polymer is a suspension of rigid particles in a Newtonian fluid (Tucker, 1991). In this case, most theories have derived the following expression for the stress tensor (Tucker, 1991):

$\boldsymbol{\sigma}=-p \boldsymbol{I}+2 \eta_{I}\left[\dot{\epsilon}+N_{p} \dot{\epsilon}: \boldsymbol{a}_{4}+N_{S}\left(\dot{\epsilon} \boldsymbol{a}_{2}+\boldsymbol{a}_{2} \dot{\epsilon}\right)\right]$

where $\eta_{I}, N_{p}, N_{s}$ are parameters depending on the fluid viscosity, fibre aspect ratio, fibre orientation and fibre concentration. They can be obtained from rheological experiments that are difficult to perform in the concentrated case. On the other hand, theoretical expressions obtained for each of these parameters depend on the concentration domain. It is most often the dilute and the semi-concentrated regime which limits greatly their wide application. Three type of approaches are used in the literature to obtain $N_{p}$ and $N_{s}$ : the slender body theory, where a particle is considered as an infinitely thin fibre (of negligible thickness) (Batchelor, 1971; Dinh and Armstrong, 1984; Shaqfeh and Fredrickson, 1990); the derivation of known results for suspensions of ellipsoidal particles, valid for finite fibre aspect ratios (Hand, 1961; Hinch and Leal, 1973; Lipscomb et al., 1988); a modification of this later model which assumes a nonlinear dependence of the effective viscosity with the fibre fraction (PhanThien and Graham, 1991). In all the above theories, $N_{p}$ is larger than $N_{s}$ for high aspect ratios. 
20.3.5.2 Slender body approximation and slender ellipsoidal particles theories for dilute and semidilute suspension.

In the approximations based on the slender body theory, $\eta_{\mathrm{I}}$ is equal to the fluid viscosity and $N_{S}=0$. They are valid for dilute (for $\phi \ll 1 / \beta^{2}$ ) (Batchelor, 1970) or for semi-concentrated suspensions (Batchelor, 1971; Dinh and Armstrong, 1984; Shaqfeh and Fredrickson, 1990). For dilute suspensions, Batchelor (1970) proposed the following expression

$N_{p}=\frac{\beta^{2} \phi}{3 \ln (\beta)}$

To extend the approach to the semi-concentrated regime, authors have considered hydrodynamic and fibre-fibre interactions, as well as lubrication forces. Hence, Batchelor (1971) gave

$$
N_{p}=\frac{\phi \beta^{2}}{9\left[\ln (2 \beta)-\ln \left(1+2 \beta \sqrt{\frac{\phi}{\pi}}\right)-1.5\right]}
$$

whereas Dinh and Armstrong (1984) considered the influence of orientation by providing $N_{p}$ as

$$
N_{p}=\frac{\phi \beta^{2}}{3 \ln \left(\frac{2 h}{D}\right)}
$$

and $h$ represents the characteristic distance between two neighbour fibres which depends on the particle orientation:

$\frac{2 h_{\text {aligned }}}{D}=\sqrt{\frac{\pi}{\phi}}$ for aligned orientation $; \frac{2 h_{\text {random }}}{D}=\frac{\pi}{2 \phi \beta}$ for random orientation

In the simulation Chung and Kwon (1996) assumed that this average distance from a given fiber to its nearest neighbours $h$ is linear in terms of the scalar measure of orientation $f_{\boldsymbol{a}_{2}}$ (defined in equation (20.20)):

$$
\begin{aligned}
& h=\left(1-f_{\boldsymbol{a}_{2}}\right) h_{\text {random }}+f_{\boldsymbol{a}_{2}} h_{\text {aligned }} \text { for } \frac{\pi}{4 \beta^{2}}<\phi<\frac{\pi}{4 \beta} \\
& h=h_{\text {aligned }} \text { for } \frac{\pi}{4 \beta}<\phi<\frac{\pi}{4}
\end{aligned}
$$

in which, the average distance between is chosen to be the same as for the aligned orientation state if fibre fraction is greater than $\pi / 4 \beta$. 
A similar expression has been obtained by Shaqfeh and Fredrickson (1990) by using a multiscattering expansion to represent the hydrodynamic interaction between fibres. Results are obtained for both random and aligned orientation states, and are found to be the same except for the numerical value of the constant, which is different for aligned orientation states and random orientation states:

$N_{p}=\frac{16 \phi \beta^{2}}{3 \ln (1 / \phi)}\left[1-\frac{\ln \ln (1 / \phi)}{\ln (1 / \phi)}+\frac{C}{\ln (1 / \phi)}\right] ; C=\left\{\begin{array}{l}0.1585 \text { (aligned fibres) } \\ 0.6634 \text { (random fibres) }\end{array}\right.$

Then, the Shaqfeh and Fredrickson theory shows a little variation of coefficient $N_{p}$ with fibre orientation. This difference with Dinh and Armstrong theory arises from the method used to calculate the cell size or the hydrodynamic screening distance: Dinh and Armstrong base their cell size on the nearest-neighbor distance, while Shaqfeh and Fredrickson use explicit modeling of the hydrodynamic interactions between multiple fibers. Finally, Ranganathan and Advani (1991) proposed a modification to the Batchelor (1970) model using a corrective factor function of the fibre aspect ratio, providing better results, but very sensitive to this correction.

Other expressions are based on ellipsoidal particles theories (Hinch and Leal, 1973; Lipscomb et al., 1988), where $N_{S}$ is different from zero but much smaller than $\mathrm{N}_{\mathrm{p}}$, and $\eta_{I}$ is different from the fluid viscosity. For dilute suspensions, they take the form:

$N_{p}=\frac{\phi}{1+2 \phi} \frac{\beta^{2}}{2[\ln (2 \beta)-1.5]} ; N_{s}=\frac{\phi}{1+2 \phi} \frac{6 \ln (2 \beta)-11}{\beta^{2}} ; \eta_{I}=\eta(1+2 \phi)$

Extensions of this model have also been proposed, to improve the results when going towards the semi-concentrated regime. In particular, Phan-Thien and Graham (1991) developed a phenomenological constitutive equation, which states that, at high volume fraction, the effective viscosity diverges and therefore they empirically got the coefficient

$N_{p}=\frac{\phi \beta^{2}}{2[\ln (2 \beta)-1.5]} \frac{\left(2-\phi / \phi_{m}\right)}{\left(1-\phi / \phi_{m}\right)^{2}} ; N_{s}=0 ; \quad \eta_{I}=\eta$

in which $\phi_{m}$ denotes the maximum volume packing. It can be evaluated by

$\phi_{m}=0.53-0.013 \beta, 5<\beta<30$ 
20.3.5.3 Model with interaction tensor for concentrated suspension

Djalili-Moghaddam and Toll (2005) hypothesised that the hydrodynamic interactions between particles in the suspension may be divided into two parts: long-range interactions which are covered by semi-dilute theory, and short-range interactions, which correspond to local lubrication forces due to the high shear rate in the gap between two nearly touching fibers. Thus, the total stress may be separated into four contributions: the static pressure, the Newtonian fluid stress, an extra stress due to fluid particle interactions and long-range particle-particle interactions, and an extra stress due to shortrange particle-particle interactions. For semi-concentrated suspensions, Férec et al. (2009) added an additional stress contribution due to the contact forces acting on the fibers. The total stress has a new term depending on the interaction tensor $\boldsymbol{b}_{4}$ and becomes:

$\boldsymbol{\sigma}=-p \boldsymbol{I}+2 \eta_{I}\left[\dot{\epsilon}+N_{p} \dot{\epsilon}: \boldsymbol{a}_{4}+N_{b} \dot{\epsilon}: \boldsymbol{b}_{4}\right]$

with

$N_{p}=\frac{\phi D^{2} X_{A}}{12 \pi} ; N_{b}=\frac{2 \phi^{2} D^{2} k}{3 \pi}$

where $X_{A}$ is the parallel drag coefficient to the fibres, dependent on the nature of the fibre-matrix contact, and $k$ is dimensionless geometric factor. For very low fibre volume fractions, $N_{b}$ becomes insignificant and the total stress reduces to the Dinh and Armstrong (1984) model.

\subsubsection{Model for non-Newtonian matrix suspension}

To our knowledge, few models in the literature take the non-linear behavior of suspending fluid into account. From the Batchelor (1971) approach (labelled as "cell model") on particles aligned in a Newtonian matrix, the first works concerning these suspensions were initiated by Goddard, (1978) for a shear-thinning fluid modelled by a power law. Following these anterious works, Souloumiac and Vincent (1998) used this method to describe the contribution of fibers to tensor of the constraints for a suspension of fibers in a power law fluid. In this approach, a cylindrical fiber of radius $R$ is included in a cell of radius $h$. The relative speed of the fluid with respect to the fiber is assumed to be undisturbed by its presence from the distance $h$. The fluid speed gradient $\nabla u$ is assumed to be constant along the 
fiber. The Souloumiac and Vincent model is based on the assumption that interactions hydrodynamics between fibers are weak. The stress tensor is then written in the following form:

$$
\begin{aligned}
\boldsymbol{\sigma}=-p \boldsymbol{I}+K \overline{\dot{\varepsilon}}^{n-1} \dot{\epsilon}+ & \frac{2 K \phi \beta^{n+1}}{n+2}\left(\frac{1-n}{n\left[1-(R / h)^{(1-n) / n}\right]}\right)^{n} \times \\
& \int|\boldsymbol{\nabla u}: \boldsymbol{p} \otimes \boldsymbol{p}|^{n-1}(\boldsymbol{\nabla u}: \boldsymbol{p} \otimes \boldsymbol{p}) \boldsymbol{p} \otimes \boldsymbol{p} \psi(\boldsymbol{p}, t) \mathrm{d} \boldsymbol{p}
\end{aligned}
$$

where $n$ is the index sensitivity to the rate of fluid deformation and $K$ its consistency. If $n$ tends towards 1, the equation is reduced to the expression of Dinh and Armstrong (Equation (20.40)). This result is also in agreement with the work of Goddard (1978) who also finds that the constraint is proportional to $\beta^{n+1}$.

Some works (Ait-Kadi and Grmela, 1994; Azaiez, 1996; Ramazani et al., 1997; Ramazani et al., 2001) concerning the modeling of the rheological properties of suspensions in viscoelastic matrices have shown that in this case the stress tensor is split as follows:

$$
\sigma=\sigma^{\mathrm{sol}}+\tau^{\mathrm{p}}+\boldsymbol{\tau}^{\mathrm{f}}
$$

in which, the contribution of the fluid to the constraint tensor of suspension is seen as the sum of the contributions due to the solvent $\boldsymbol{\sigma}^{\mathrm{sol}}$ and the polymer $\boldsymbol{\tau}^{\mathrm{p}}$ and the fiber contribution $\boldsymbol{\tau}^{\mathrm{f}}$ is computed by taking the whole viscosity (solvant and polymer) in order to take into account of non-newtonian input.

The equation general form of stress tensor (20.37) induces that the viscosity of fiber suspension is anisotropic and therefore can be express as a fourth-order tensor. Expressions of these fourth-order viscosity tensors are proposed by looking at particular configurations (Sommer et al., 2018; Wittemann et al., 219;. More recently, (Li and Luyé, 2019; Favaloro et al., 2018; Tseng and Favaloro, 2019) have proposed to replace this fourth-order viscosity tensor by an optimal scalar model which is implemented in both Moldflow Insight API framework and Moldex3D software.

20.4 Computation of fibre orientation in injection moulding

We present computational examples of fibre orientation in injection moulding for which experimental data exist. The various moulds are geometrically complex enough to describe problems encountered in 
real industrial processes. In the first example, we check whether the skin-core effect is accurately computed in a rectangular plaque. Moreover, the influence of the orientation-rheology coupling and of the interaction coefficient $C_{I}$ is analysed by computing the orientation near the inlet gate. The second example is a U-shape with thin walls and ribs which is used in the automotive industry showing that we are able to compute the appearance and location of weld lines. The third example is the plaque with seven different ribs (see Figure 20.2) which gives information on the orientation state in small parts.

First of all, the numerical methods are briefly described. The methodology is that used in Rem3D software and it is based on an Eulerian approach (the whole injection cavity is meshed) and a stabilised Galerkin method, using a continuous approximation of the orientation tensor coupled to flow (Generalized Stokes behaviour), the thermal equation, the flow front evolution (using a level set method) and fibre orientation equations (Silva et al., 2016).

\subsubsection{Numerical methods}

A weakly coupled approach is used meaning that the problem is solved in two steps. This weakly coupling did not need internal loop to achieve convergence between velocity and orientation computations. Indeed, the computation of the moving fluid/air interface (equation (20.54)) imposed a rather small time step which has made it unnecessary. The first step involves the solution of the mechanical problem, assuming that the polymer is incompressible and neglecting gravity and inertia:

$\nabla \cdot \sigma=0$ and $\nabla \cdot v=0$

with an isotropic orientation at the first step or an orientation tensor determined at the previous time step for the other instants. Its contribution is taken in account thanks to equation (20.37) and the fourth- order tensor is approximated with a quadratic relation (20.17). A classical mixed finite element method (Pichelin and Coupez, 1988) is used, in which the orientation tensor is implicitly considered.

The fluid is supposed to follow an incompressible shear-thinning behaviour, represented by a CarreauYasuda and Arrhenius laws for the viscosity $\eta$ : 
$\eta=\eta_{0}(T)\left[1+\left(\eta_{0}(T) \frac{\dot{\bar{\epsilon}}}{\tau_{c}}\right)^{\alpha}\right]^{\frac{m-1}{\alpha}}$ and $\eta_{0}(T)=\eta_{0}\left(T_{\mathrm{ref}}\right) \exp \left(\beta_{T}\left[\frac{1}{T}-\frac{1}{T_{\mathrm{ref}}}\right]\right)$

The parameters occurring in these laws are given in Tables 0.2 for the fluid studied

As viscosity depends on temperature, the energy equation resolution is coupled and solved:

$\rho C_{p}\left(\frac{\partial T}{\partial t}+v \cdot \nabla T\right)=\nabla \cdot\left(k_{T} \nabla T\right)+\eta \dot{\bar{\epsilon}}^{2}$

where $\rho$ is the volume density, $C_{p}$ the specific heat, $k_{T}$ the thermal conductivity, and the last term represents the viscous dissipation. These parameters are considered to be constant. Finally, heat conduction towards the mould walls and viscous heating are also taken into account.

The second step involves the computation of fibre orientation with velocity field obtained in the previous step, through the resolution of evolution equation (20.16). Numerical resolution of Folgar and Tucker's equation has been performed by Kabanémi and Hétu (1999) using the fourth order Runge-Kutta method, by Martinéz et al. (2003) with the method of characteristics, by Pichelin and Coupez (1999) and Redjeb et al. (2005) with a space-time discontinuous Galerkin scheme. Miled et al. (2008) have proposed a standard Galerkin method associated to a RFB or SUPG stabilisation, which prevents inaccurate oscillations due to the hyperbolic character of this equation. Moving interface (such as fluid/air) is also calculated at each time step by solving a convection equation (Ville et al., 2011) associated to a signed distance function which defines the fluid domain (its value is positive in the fluid and negative in the empty region):

$\frac{\partial \alpha}{\partial t}+\boldsymbol{v} \cdot \nabla \alpha=0$

Finally, all parameters and equations are extended to the whole computational domain, as the weak formulation of the finite element method ensures the continuity of stress, velocity, orientation tensor and temperature (Batkam et al., 2004).

The numerical approach detailed here is largely used in multiphysics, multidomain and multiphase modeling of composites as summarized in Silva et al. (2016). Theses advanced numerical techniques are based on mixed stabilized finite elements, Eulerian approaches and modified level-set techniques 
(Nakhoul et al., 2018), automatic anisotropic mesh adaptation and parallel computing (Digonnet et al., 2019).

\subsubsection{Representation of orientation}

The problem is now to get practical information from the computed orientation tensor. In an orthonormal Cartesian basis, the diagonal term $a_{i i}$ of the orientation tensor quantifies the fibre alignment along the main axis. As already mentioned in the first section, $a_{11}=1$ means that all the fibres are oriented along the direction $\boldsymbol{i}_{1}$. Moreover, $a_{11}=0$ indicates that all the fibres are perpendicular to the axis $\boldsymbol{i}_{1}$, that is, they belong to the plane $\left(\boldsymbol{i}_{2}, \boldsymbol{i}_{3}\right)$. The components $a_{i j}$ with $i \neq j$ quantify the asymmetry of the distribution of orientation relative to the directions $\boldsymbol{i}_{i}$ or $\boldsymbol{i}_{j}$. For isotropic orientation in 3D, the components are $a_{i i}=1 / 3$ while $a_{i j}=0$ for $i \neq j$.

Another way to describe the computed orientation is to plot the ellipsoid associated to the eigenvalues, $\Lambda_{i}$, and eigenvectors, $\mathbf{e}_{i}$, of the orientation tensor $\mathbf{a}_{2}$ (Advani and Tucker,1987) (Altan et al., 1990). The eigenvectors and eigenvalues of the orientation tensor give, respectively, the direction and length axes of the ellipsoid as shown in figure 20.6.

In order to determine the degree of anisotropy, a Von Mises effective orientation, $a_{v}$, can also be computed:

$a_{v}=\sqrt{\frac{\left(a_{11}-a_{22}\right)^{2}+\left(a_{22}-a_{33}\right)^{2}+\left(a_{11}-a_{33}\right)^{2}+6\left(a_{12}^{2}+a_{13}^{2}+a_{23}^{2}\right)}{2}}$

It is null for an isotropic orientation and equals to 1 if all fibres are oriented along a principal direction.

20.4.3 Rectangular plaque with inlet gate 
We consider the injection moulding of a three-dimensional plaque (figure 20.8), and in particular we study the orientation development near the injection gate. Dimensions of the plaque are given in figure 20.7. Only half of the plaque will be considered in the simulation, since the geometry presents a symmetry plane.

The polymer is a polyarylamide (Solvay Ixef 1022$)$ reinforced with $50 \%$ weight ( $31.6 \%$ volume) glass fibres. The fibre aspect ratio is considered constant and equal to 10 . The injection is done at a flow rate of $20 \mathrm{~cm}^{3} / \mathrm{s}$ and the initial polymer temperature is $270{ }^{\circ} \mathrm{C}$. The mould temperature is kept constant at $130{ }^{\circ} \mathrm{C}$, and the mould is filled in $2.70 \mathrm{~s}$. The computational time step is $0.002 \mathrm{~s}$. The orientation distribution is analysed after 1300 time iterations, almost at the end of cavity filling.

In order to reduce the computational time, the long circular channel has not been taken into account. The inlet of the cavity is just after the last curve of the feeding channel. The computations are made on a half mould with symmetric boundary conditions on the plane $\left(\boldsymbol{i}_{1}, \boldsymbol{i}_{2}\right)$. The problem is now to impose an initial orientation at the cavity inlet as we do not take into account the cylindrical channel. Usually one considers an isotropic initial orientation. But the flow in the channel is dominated by shear deformations, which are known to orient fibres mainly in the flow direction (namely the $\boldsymbol{i}_{1}$ axis) at the entrance of the triangular gate. Nevertheless, the two curves in the cylindrical channel can disturb this orientation. So, computations are made for two different initial conditions (isotropic case, $a_{i i}=1 / 3$, and unidirectional case, $a_{11}=1$ ) and a hybrid closure approximation. Moreover, two values of the interaction coefficient $\left(C_{I}=0.001\right.$ and 0.04$)$ and two values of the rheological coupling coefficients $\left(N_{p}=0,100\right.$ and $\left.N_{s}=0\right)$ will be tested.

Figures 20.7 show cross sections in the $\left(\boldsymbol{i}_{1}, \boldsymbol{i}_{2}\right)$ plane at the end of filling and the distribution of the diagonal orientation tensor components. We notice that the tensor is almost diagonal, so the $\left(\boldsymbol{i}_{1}, \boldsymbol{i}_{2}, \boldsymbol{i}_{3}\right)$ axes are the principal axes of the orientation tensor. Also, the skin-core effect initiated at the end of the gate is preserved until the end of the plaque. Computed values of $a_{22}$ are very small, except at the junction between the divergent region and the plaque. This means that fibres are parallel to the $\left(\boldsymbol{i}_{1}, \boldsymbol{i}_{3}\right)$ plane in the areas where experimental observations are made. This is in agreement with the observations and we will focus on the component $a_{11}$ which quantifies the degree of alignment 
with the flow direction. The triangular gate region is divergent in the plane $\left(\boldsymbol{i}_{1}, \boldsymbol{i}_{3}\right)$ and convergent in the plane $\left(\boldsymbol{i}_{1}, \boldsymbol{i}_{2}\right)$ (see figure 20.7). The negative elongation rate along the $\boldsymbol{i}_{3}$ axis dominates over the positive one along $\boldsymbol{i}_{1}$ axis and an orientation perpendicular to the $\boldsymbol{i}_{1}$ direction develops in the core. This orientation is transported at the junction between the gate and the plaque so that at the entrance of the plaque, we obtain a skin-core orientation, with a skin oriented in the flow direction, and a core perpendicular to it. These calculations show how a gate can lead to a skin-core structure in moulded parts. The computations are qualitatively in agreement with the observations.

In figures 20.9, the evolution of $a_{11}$ with $x_{2}$, the plaque thickness, is shown at the four positions A, B, C and D where the experimental measurements were made (see figure 20.7b). Computations were performed with an isotropic initial orientation showing that:

1. A skin-core structure is rapidly formed in zone A, and it remains even in D after the junction gateplaque. As shown in the experiments, the skin/core structure is less significant for position $\mathrm{C}$ than for position D;

2. For a partially coupled calculation $\left(N_{p}=0\right)$, when the interaction coefficient $C_{I}$ increases, near the surface, fibres are less oriented in the flow direction. For example, in the first position A for the lower wall $\left(x_{2}=2.5 \mathrm{~mm}\right)$, one gets $a_{11} \sim 0.7$ for $C_{I}=0.04$ and $a_{11} \sim 0.9$ for $C_{I}=0.001$. This can be explained by the fact that shear is dominant near the walls, and it tends to orient fibres in the flow direction. The interaction term tends to disorient fibres, and its effect increases with $C_{I}$. The orientation in the core does not really change when $C_{I}$ varies;

3. An increase of $N_{p}$ from 0 to 100 (that is comparison between coupled and partially coupled calculations) leads to complex tendencies in the skin regions. In the lower skin region, that is for $x_{2}=2.5 \mathrm{~mm}, a_{11}$ decreases in position $\mathrm{A}$ and $\mathrm{B}$, but the change is negligible in positions $\mathrm{C}$ and D. At the opposite skin, there is no noticeable effect for positions B and D. The trends are opposite in region $\mathrm{A}\left(a_{11}\right.$ increases $)$ and $\mathrm{C}\left(a_{11}\right.$ decreases $)$; that phenomenon points out the complex coupling effect on the flow velocity. 
4. In the core region, taking into account the rheological coupling leads to a small increase of $a_{11}$, except in position $\mathrm{D}$ for $C_{I}=0.04$. In position $\mathrm{D}$, the lowest value of $a_{11}$ is for $N_{p}=100$ and $C_{I}=0.04$

Computations realised with unidirectional initial orientation (see figures 20.10) show that:

1. For the weakly coupled calculations $\left(N_{p}=0\right)$ and $C_{I}=0.04$, a skin/core structure is formed as for the isotropic initial orientation. Nevertheless, the core is less oriented perpendicular to the flow direction: we get $a_{11}$ around $0.3-0.4$ for positions $\mathrm{A}, \mathrm{B}, \mathrm{C}$, instead of $0.2-0.3$. The behaviour is completely different for $C_{I}=0.001$ : orientation is in the flow direction on the whole thickness at positions A and B. $a_{11}$ begins to decrease a little in the core in position C, and in $\mathrm{D}$ the orientation is isotropic $\left(a_{11}=0.5\right)$;

2. when $N_{p}$ goes from 0 to 100 , in the skin and core regions, $a_{11}$ decreases a little, or remains nearly constant, depending on the position and the value of $C_{I}$;

3. The influence of the initial orientation is not very important in the last probe $\mathrm{D}$, especially at the surfaces, except for $C_{I}=0.001$ and $N_{p}=0$ in the core: orientation is $2 \mathrm{D}$ isotropic in plane $\left(\boldsymbol{i}_{1}, \boldsymbol{i}_{2}\right)$ for a unidirectional initial orientation $\left(a_{11}=0.25\right)$, whereas a transverse orientation is formed with an isotropic initial orientation $\left(a_{11}=0.25\right)$.

Numerical results have been compared to the experiments (Redjeb, 2007). The measured points are in between the results obtained with both initial orientation, but the agreement is better for the unidirectional initial orientation. This is not surprising, as a circular channel orients fibres mainly in the flow direction. In the skin regions, the agreement is quite good with $C_{I}=0.04$ for the four locations A, B, C and D. The coupled calculation does not improve significantly the quality of the agreement. In the core region, agreement is better for $C_{I}=0.001$. These results show that the interaction coefficient may depend on the orientation. However, the computed skin/core orientation at the beginning of the plaque remains more important with respect to the measurements.

20.4.5 U-shaped part with thin walls and cross ribs 
The studied part is presented in figure 20.12a. The computations have used a mesh generated by a topologic mesher (Gruau and Coupez, 2005) or (Coupez, 2011) governed by a natural metric in order to capture the thin walls. This metric takes into account, for each area of mould, a specified mesh size in each principal direction ensuring an appropriate number of elements in the whole thickness of the part. The injected material is a polypropylene referenced as STAMAX P30YM240. This material is also modelled by a Carreau-Yasuda law for the rheology and an Arrhenius law for thermo-dependency (the parameters are given in table 20.3). The polymer is injected at $250^{\circ} \mathrm{C}$ and we assume that the mould is fully regulated at $40^{\circ} \mathrm{C}$. Initially, the air inside the cavity is also considered at a temperature of $40^{\circ} \mathrm{C}$.

To compute the fibre orientation, one takes a fibre aspect ratio $\beta=10$, an interaction coefficient $C_{I}=$ 0.001 , a hybrid closure approximation and a weakly coupling, $N_{p}=0$. The orientation is analysed after a total filling time of $3.7 \mathrm{~s}$ (the time step is $310^{-4} \mathrm{~s}$ ). The dynamics of filling is important because it helps to understand the dynamics of fibre orientation. The flow is fully symmetrical and it starts from inlet to fill the bottom, the walls and the crossing central ribs simultaneously. At the beginning, the wall filling is slightly delayed compared to central ribs. This delay disappears and is reversed as soon as the cavity is half filled.

Finally, the part having cross-ribs has two weld lines as the material fronts flowing in the flank and ribs intersect twice. The first weld line is located on the sidewall after crossing the first rib. The second weld line is located at the second row of crossing ribs, as the flow front from the sidewall is faster and has time to penetrate the rib.

We focus now on the first weld line which is easily detected by the computation. Figure $20.12 b$ is photography of part which shows the weld line. The injection gate is located at the bottom right and the presence of fibres at the surface allows seeing this line which is located in the middle of wall. This line is slightly oriented in the direction of flow.

Tracing the isosurfaces of components of the orientation tensor (see figure 20.13), it is possible to find this weld line. The orientation on the side wall becomes unidirectional along the $\boldsymbol{i}_{1}$ direction: $a_{11}$ increases while $a_{22}$ remains relatively low. At the intersection of the two fronts in the region where the 
ribs are connected to the wall, we can observe a decrease of component $a_{11}$ along the entire weld line and an increase of component $a_{22}$. Therefore, the weld line is well located and predicted where there is a disturbance of the orientation tensor.

20.4.5 Square plaque with ribs

The studied part is presented in figure 20.2. It is a plaque with seven ribs and it is filled from one side. The variation of fibre concentration inside the part described in the second section is not considered in our model although it is important in terms of mechanical properties. The part has a plane of symmetry and computations are made only on half of the cavity. These computations are made for aspect ratio $\beta=30, C_{I}=0.023$ and $N_{S}, N_{p}=0$. The fluid is a Stamax P40YM243 composed by a Polypropylene matrix and $40 \%$ of glass fibres. The total filling time is around $1 \mathrm{~s}$. Finally computations are compared to experimental observations.

In figures 20.14 and 20.15, the ellipsoids associated to orientation tensor are plotted and they are more or less elongated according to fibre orientation. They are also coloured by von Mises scalar (equation (20.55)) in order to give information on the anisotropy of fibre orientation. In figure 20.12, the skincore phenomenon is observed in plaque as shown in experiments. However, with the Folgar and Tucker model, an isotropic orientation is obtained in the core instead of an orientation perpendicular to the flow motion in the experiment. In the skin region, the orientation along the flow motion is observed both in experiments and computations.

For the ribs, the orientation changes during filling: first during rib filling, a core/skin orientation is observed; secondly, there is a "disorientation" of fibres once the rib is filled. Finally only the base of the rib maintains a non-isotropic orientation.

In figure 20.14, we compare the simulation with the experimental observation when the mould is fully filled (experimental picture is slightly larger than the image obtained by the simulation).

We deduce that the simulation allows reproducing the increase of the thickness of core area at the base of the ribs. Moreover, the numerical model reproduces the arch drawn orientation. As in experimental observations, a homogeneous orientation is recovered inside the rib. However, this orientation is isotropic in simulations, whereas the fibres are oriented in a direction parallel to the rib in experiments. 
Moreover, the arch orientation depends on the time of packing which was not taking into account in our simulations.

Finally, Rem3D software recovers reasonably faithfully the orientation encountered in the experiments. Only the areas having an isotropic orientation are not in agreement with the experiments. To overcome this discrepancy, a tensorial interaction coefficient $C_{I}$ has to be introduced as prescribed by anisotropic rotary diffusive models. In this way, the anisotropy of the plaque will be taken into account (the vertical direction is smaller with respect to the two others).

\subsection{Conclusions}

Fibre structure in reinforced moulded components is directly related to the process. Fibre length degradation takes place mostly in the plasticising unit but the fibre length distribution is not yet considered in our modelling. Fibre concentration can be considered as constant in first approximation even though, in the plaque with ribs, we measured some important fluctuations inside the part. Therefore the evolution of fibre concentration may have an influence on the evolution of fibre orientation during the process. Fibre orientation varies a lot throughout the part, especially in the thickness. A core region with fibres transverse to the flow direction is almost always created in the gate region, because of high elongation rates. Near the surfaces, fibres are predominantly oriented in the flow direction.

Quantification of fibre orientation is time consuming, with several causes of errors. Modelling of flowinduced fibre orientation is usually carried out supposing an equivalent viscous behaviour for the composite. This gives valuable information for mould design, and especially gate location. The closure approximation has a larger influence on the results than does the interaction coefficient. Full 3D computations give a precise kinematics description in the gate, at the flow front, which increases the precision of the orientation calculation. Coupling between rheology and orientation becomes important, raising the issue of the validity of constitutive equations based on dilute or semi-dilute Newtonian suspension, and of the determination of rheological parameters. The numerical computations describe reasonably well the orientation encountered in the experiments. The 
discrepancy could be overcome by making the model more complex (as the RSC model described in section 20.3.3), but the number of parameters increases and there is no simple way to get them. Then, both the experimental setup and numerical procedures (inverse analysis) have to be developed to identify these parameters for the polymers used in industrial process. For example, a systematic procedure to minimize the discrepancy between the numerically predicted and the experimentally measured fiber orientation results on an injection-molded part is proposed in ( $\mathrm{Li}$ and Luyé, 2018) 
Autodesk Help, Moldflow Rotational Diffusion (MRD) Model,' (2018), https://knowledge.autodesk.com/support/moldflow-insight/downloads/caas/CloudHelp/ cloudhelp/2019/ENU/MoldflowInsight-Analyses/files/GUID-54ED8884-6EB2-44B9-98F49E6817CBF6F5-htm.html

Advani S G, Tucker C L (1987), 'The use of tensors to describe and predict fibre orientation in short fibre composites', $J$ Rheology, 31, 751-784.

Advani S G, Tucker C L (1990), 'Closure approximations for three-dimensional structure tensors', $J$ Rheol, 34, 367-386.

Ait-Kadi A and Grmela M (1994). 'The rheological behaviour of fibre suspensions in viscoelastic media', J. Non-Newtonian Fluid Mech., 53, 65-81.

Akay M, Barkley D (1991) 'Fibre orientation and mechanical behaviour in reinforced thermoplastic injection moldings', $J$ Mat Sci, 26, 2731-2742.

Akay M, O'Regan D F, Bailey R S (1995), 'A model to predict the through-thickness distribution of heat generation in cross-ply carbon-fibre composites', Comp Sci Technol, 55, 109-118.

Altan M C, Selcuk S S, Guceri I, Pipes R B (1990), 'Numerical prediction of three-dimensional fibre orientation in hele-shaw flows', Polym Eng Sci, 30, 848-859.

Anczurowski E, Mason S G (1968), 'Particle motions in sheared suspensions. XXIV. Rotation of rigid spheroids and cylinders', Trans Soc Rheol, 12, 209-2015.

Avérous L, Quantin J C, Crespy A, Lafon D (1997), 'Evolution of the three-dimensional orientation of glass fibres in injected isotactic polypropylene', Polym Eng Sci, 37(2), 329-337.

Azaiez J (1996), 'Constitutive equations for fiber suspensions in viscoelastic media', $J$. Non-Newtonian Fluid Mech. 66, 35-54.

Bakharev A, Yu H, Ray S, Speight R, Wang J (2018), 'Using new anisotropic rotational diffusion model to improve prediction of short fibers in thermoplastic injection molding', in: SPE ANTEC Conference, Orlando, FL.

Batchelor G K (1970), 'Slender body theory for particles arbitrary cross section in stokes flow', $J$ Fluid Mech, 44, 419-440.

Batchelor G K (1971), 'The stress generated in a non-dilute suspension of elongated particles by pure straining motion', J Fluid Mech, 46, 813-829

Batkam S, Bruchon J, Coupez T (2004), 'A space-time discontinuous Galerkin method for convection and diffusion in injection moulding', Int J Forming Processes, 7, 11-33

Bay R S, Tucker III C L (1992), 'Stereological measurement and error estimates for three-dimensional fibre orientation', Polym Eng Sci, 32(4), 240-253

Beaume G (2008), 'Modelling and numerical simulation of a complex fluid flow', PhD thesis ENSMP, http://tel.archives-ouvertes.fr/tel-00416435/fr/ 
Binder A (1939), 'The motion of cylindrical particles in viscous flow', J Appl Phys, 10, 711.

Bird R B, Armstrong R C, Hassager O (1987), 'Dynamics of Polymeric Liquids. Volume 1, Fluid Mechanics', Wiley, New York.

Bird R B, Curtiss C F, Armstrong R C, Hassager O (1987), 'Dynamics of Polymeric Liquids. Volume 2, Kinetic Theory', Wiley, New York.

Borzacchiello D, Abisset-Chavanne E, Chinesta F, Keunings R (2016), 'Orientation kinematics of short fibres in a second-order viscoelastic fluid', Rheol Acta, 55, 397-409.

Bretherton F P (1962), 'The motion of rigid particles in a shear flow at low Reynolds number', $J$ Fluid Mech, 14, 284-304.

Brunn P (1977), 'The slow motion of a rigid particle in a second-order fluid', J Fluid Mech, 82, 529547.

Chin W, Liu H, Lee Y (1988), 'Effects of fibre length and orientation distribution on the elastic modulus of short fibre reinforced thermoplastics', Polym Comp, 9(1), 27-35.

Chung D H, Kwon T H (1996), 'Coupled Analysis of Injection Molding Filling and Fiber Orientation, Including In-Plane Velocity Gradient Effect', Polym Compos, 17(6), 859-872.

Chung D H, Kwon T H (2001), 'Improved model of orthotropic closure approximation for flow induced fibre orientation', Polym Compos, 22(5), 636-649.

Chung D H, Kwon T H, (2002a), 'Invariant-based optimal fitting closure approximation for the numerical prediction of flow-induced fibre orientation, J.Rheol. 46(1), 169-194.

Chung D H, Kwon T H (2002b), 'Fibre orientation in the processing of polymer composites', KoreaAustralia Rheology Journal, 107, 67-96.

Cintra J, Tucker C L (1995), 'Orthotropic closure approximation for flow induced fibre orientation', $J$ Rheol, 34, 1095-1122.

Clarke A, Eberhardt C (2002), Microscopy techniques for materials science, Woodhead Publishing, Cambridge.

Coupez T (2011), 'Metric construction by length distribution tensor and edge based error for anisotropic adaptive meshing', J Comp Phys, 230(7), 2391-2405.

Cox R G (1971), 'The motion of long slender bodies in a viscous fluid. Part 2: shear flow', J Fluid Mech, 45, 625-657.

D'Avino G, Hulsen M, Greco F, Maffettone P (2014), 'Bistability and metabistability scenario in the dynamics of an ellipsoidal particle in a sheared viscoelastic fluid', Phys Rev E, 89, 043006.

Davidson N C, Clarke A R (1999), 'Extending the dynamic range of fibre length and fibre aspect ratios by automated image analysis', J Microscopy, 196, 266-272.

Denault T, Vu-Khanh T, Foster B (1989), 'Tensile properties of injection molded long fibre thermoplastic composites. Polymer Composites', Polym Compos, 10, 313-321.

Diamant H (2009), 'Hydrodynamic interaction in confined geometries', J Phys Soc Jpn, 78, 041002. 
Digonnet H, Coupez T, Laure P, Silva L (2019), 'Massively parallel anisotropic mesh adaptation', Int J High Performance Computing Applications, 33(1), 3-24.

Dinh S, Armstrong R (1984), 'A rheological equation of state for semi-concentrated fibre suspensions', J Rheol, 28, 207-227.

Djalili-Moghaddam M, Toll S (2005), 'A model for short-range interactions in fibre suspensions', $J$ Non-Newtonian Fluid Mech, 132, 73-83.

Dontula N, Ramesh N S, Campbell, G A, Small J D, Fricke A L (1994), 'An experimental study of polymer-filler redistribution in injection molded parts', J Reinf Plast Compos, 13, 98-110.

Doi M, Edwards S F (1986), 'The Theory of Polymer Dynamics', Oxford Science Publications, NewYork

Dupret F, Verleye V (1999), 'Modeling the flow of fibre suspensions in narrow gaps', in Rheology Series 8, Siginer D A, De Kee D, Chhabra R P (eds), Elsevier, Amsterdam, 1347-1398.

Faessel M, Delisée C, Bos F, Castera P (2005), 3D modelling of random cellulosic fibrous networks based on X-ray tomography and image analysis, Comp. Sci Technol, 12, 1931-1940.

Fan X, Phan-Thien N, Zheng R (1998), ‘A direct simulation of fibre suspensions', J Non-Newtonian Fluid Mech, 74, 113-135. 
Favaloro A J, Tseng H-C, and Pipes R B (2018) 'A new anisotropic viscous constitutive model for composites molding simulation', Compos. Part A: Appl. Sci. Manuf. 115, 112-122.

Favaloro A J, Tucker III C L (2019), 'Analysis of anisotropic rotary diffusion models for fibre orientation', Composites Part A: Applied Science and Manufacturing, 126, 105605.

Férec J, Ausias G, Heuzey M C, Carreau P J (2009), 'Modeling fibre interactions in semi-concentrated fibre suspensions', $J$ Rheol, 53, 49-72.

Férec J, Abisset-Chavanne E, Ausias G, Chinesta F (2014), 'On the use of interaction tensors to describe and predict rod interactions in rod suspensions', Rheologica Acta, 53, 445-456.

Férec J, Ausias G (2015), 'Rheological Modeling of Non-dilute Rod Suspensions', In Rheology of Non-Spherical Particle Suspensions, Chinesta F, Ausias G (eds), Elsevier, 77-117.

Férec J, Perrot A, Ausias G (2015), 'Toward modeling anisotropic yield stress and consistency induced by fibre in fibre-reinforced viscoplastic fluids', J Non-Newtonian Fluid Mech., 220, 69-76.

Férec J, Bertevas E, Khoo B C, Ausias G, Phan-Thien N (2016), 'The effect of shear-thinning behaviour on rod orientation in filled fluids', J Fluid Mech, 798, 350-370.

Folgar F, Tucker C L (1984), 'Orientation behaviour of fibres in concentrated suspensions', J Reinf Plast Compos, 3, 98-119.

Franzen B, Klason C, Kubat J, Kitano T (1989), 'Fibre degradation during processing of short fibre reinforced thermoplastics. Composites', Composites, 20(1), 65-76.

Goddard J. D. (1978), 'Tensile behavior of power law fluids containing oriented slender fibers'. $J$. Rheol., 22, 615-622.

Gruau C, Coupez T (2005), '3D tetrahedral, unstructured and anisotropic mesh generation with adaptation to natural and multidomain metric', Comp Meth Appl Mech Eng, 194 , 4951-4976.

Guiraud O, Orgéas L, Dumont P J J, Rolland du Roscoat S (2012), 'Microstructure and deformation micro-mechanisms of concentrated fibre bundle suspensions: an analysis combining x-ray microtomography and pull-out tests', J Rheol, 56, 593-623.

Gunes D Z, Scirocco R, Mewis J, Vermant J (2008), 'Flow-induced orientation of non-spherical particles: effect of aspect ratio and medium rheology', J Newt Fluid Mech, 155, 39-50.

Gupta V B, Mittal R K, Sharma P K, Menning G, Wolters J (1989), 'Some studies on glass fibrereinforced polypropylene. Part II: Mechanical properties and their dependence on fibre length, interfacial adhesion and fibre dispersion', Polym Comp, 10(1), 8-15.

Hand G (1961), 'A theory of dilute suspension', Arch Rat Mech Anal, 7, 81-86.

Hand G L, 'A Theory of Anisotropic Fluids', J Fluid Mech, 13, 1962, 33-46.

Hegler R P, Menning G (1985), 'Phase separation effects in the processing of glass bead and glass fibre filled thermoplastics by injection molding', Polym Eng Sci, 25, 395-405.

Hinch E, Leal L (1973), 'Time dependent shear flows of a suspension of particles with weak Brownian rotations', J Fluid Mech, 57, 753-767. 
Iso Y, Koch D L, Cohen C (1996), 'Orientation in simple shear flow of semi-dilute fibre suspensions. 1. Weakly elastic fluids', J Newt Fluid Mech, 62, 115-134.

Iso Y, Cohen C, Koch D L (1996), 'Orientation in simple shear flow of semi-dilute fibre suspensions. 2. Highly elastic fluids', J Newt Fluid Mech, 62, 135-153.

Jeffery G B (1922), ‘A theory of anisotropic fluids', Proc Roy Soc London, A102, 161-179.

Kabanémi K K, Hétu J F (1999), 'Modelling and simulation of nonisothermal effects in injection moulding of rigid fibres suspensions', J Inj Molding Technol, 3, 80-87.

Kamal M R, Song L, Singh P (1986), 'Measurement of fibre and matrix orientations in fibre reinforced composites', Polym Comp, 7(5), 323-832.

Koch D L (1995), ‘A model for orientational diffusion in fibre suspensions', Phys Fluids, 7, 20862088.

Kubat J, Szalanczi A (1974), 'Polymer-Glass Separation in the Spiral Mold Test', Polym Eng Sci, 14, 873-877.

Latil P, Orgéas L, Geindreau C, Dumont P J J, Rolland du Roscoat S (2011), 'Towards the 3d in situ characterisation of deformation micro-mechanisms within a compressed bundle of fibres', Compos Sci Technol, 71, 480-488.

Laurencin L. (2017), '3D Micro-Rheology of non-Newtonian fibre suspensions using fast X-ray tomography and Finite Element simulations at fibre scale', PhD thesis Grenoble University, https://tel.archives-ouvertes.fr/tel-01688286

Laurencin T, Orgéas L, Dumont P J J, Rolland du Roscoat S, Laure P, Le Corre S, Silva L, Mosko R, Terrien M (2017), '3D real-time and in situ characterization of fibre kinematics in dilute nonNewtonian fibre suspensions during confined and lubricated compression flow', Compos Sci Tech, $134,258-266$.

Laurencin T, Laure P, Orgéas L, Dumont P J J, Silva L, Rolland du Roscoat S (2019), 'Fibre kinematics in dilute non-Newtonian fibre suspensions during confined and lubricated squeeze flow: Direct numerical simulation and analytical modelling', J Non-Newt Fluid Mech, 273, 104187.

Le T H, Dumont P J J, Orgéas L, Favier D, Salvo L, Boller E (2008), 'X-ray phase contrast microtomography for the analysis of the fibrous microstructure of SMC composites', Compos Part A, $39,91-103$.

Leal L (1975), 'The slow motion of slender rod-like particles in a second-order fluid', J Fluid Mech, $69,305-337$.

Li T and Luyé J-F (2018), 'Optimization of Fiber Orientation Model Parameters in the Presence of Flow-Fiber Coupling', J. Compos. Sci. 2, 73-90

T. Li and J.-F. Luyé (2019), 'Flow-Fiber Coupled Viscosity in Injection Molding Simulations of Short Fiber Reinforced Thermoplastics', International Polymer Processing 34(2), 158-171. 
Limodin N, Salvo L, Boller E, Suery M, Felberbaum M, Gailliegue S, Madi K (2009), 'In situ and real-time 3-d microtomography investigation of dendritic solidification in an ale-10 wt.\% cu alloy', Acta Mater, 57, 2300-2310.

Lipscomb G G, Keunigs R, Marucci G, Denn M M (1984), 'A continuum theory for fibre suspensions', Proc. IX Int. Congress on Rheology, 2, 497-503.

Lipscomb G G, Denn G, Hur M, Hur D, Boger D (1988), 'The flow of fibre suspensions in complex geometries', J Non-Newtonian Fluid Mech, 26, 297-325.

Megally A (2005), 'Étude et modélisation de l'orientation de fibres dans des thermoplastiques renforcés', PhD Thesis ENSMP, https://tel.archives-ouvertes.fr/pastel-00001471

Mezher R, Perez M, Scheuer A, Abisset-Chavanne E, Chinesta F, Keunings R (2016), 'Analysis of the Folgar \& Tucker model for concentrated fibre suspensions in unconfined and confined shear flows via direct numerical simulation', Composites: Part A, 91, 388-397.

Martinéz M A, Cueto E, Doblaré M, Chinesta F (2003), 'Natural element meshless simulation of flows involving short fibre suspensions', $J$ Non-Newtonian Fluid Mech, 115, 51-78.

Miled H, Silva L, Agassant J F, Coupez T (2008), 'Numerical simulation of fibre orientation and resulting thermo-elastic behaviour in reinforced thermoplastics', in Mechanical Response of Composites, Camanho P P, Dávila C G, Pinho S T, Remmers J J C (eds), Springer, Berlin, 293-313.

Moses K B, Advani S G, Reinhardt A (2001), 'Investigation of fibre motion near solid boundaries in simple shear flow', Rheol Acta, 47, 63-73.

Nakhoul R, Laure P, Silva L, Vincent M (2018), 'A multiphase Eulerian approach for modelling the polymer injection into a textured mould', Int J Material Forming, 11, 53-66.

Onsager L (1949), 'The effects of shape on the interaction of colloidal particles', Ann NY Acad Sci, 51, 627-659.

Orgéas L, Dumont P J J, Vassal J P, Guiraud O, Michaud V, Favier D (2012), 'In-plane conduction of polymer composites plates reinforced with architectured networks of copper fibres', J Mater Sci, 47, 2932-2942.

Ozolins A, Strautins U (2014), 'Simple models for wall effect in fibre suspension flows', Math. Model. Anal, 19, 75-84.

Papathanasiou T D (1997), 'Flow-induced alignment in injection molding of fibre-reinforced

Papathanasiou T D (1997), 'Flow-induced alignment in injection molding of fibre-reinforced polymer composites', in Flow-induced alignment in composite materials, Papathanasiou T D and Guell D C (eds), Woodhead Publishing, Cambridge, UK, 112-165.

Petrie C J S (1999), ‘The Rheology of Fibre Suspensions', J Non-Newtonian Fluid Mech, 87, 369-402.

Petrich M P, Koch D L, Cohen C (2000), 'An experimental determination of the stress microstructure relationship in semi-concentrated fibre suspensions', J Newt Fluid Mech, 95, 101-133.

Perez M, Scheuer A, Abisset-Chavanne E, Chinesta F, Keunings R (2016), 'A multiscale description of orientation in simple shear flows of confined rod suspensions', J Newt Fluid Mech, 233, 61-74. 
Phan-Thien N, Graham A L (1991), 'A New Constitutive Model for Fibre Suspensions: Flow Past a Sphere', Rheologica Acta, 30, 44-57.

Phan-Thien N, Fan X J, Tanner R I, Zheng R (2002), 'Folgar-Tucker constant for a fibre suspension in a Newtonian fluid', J Non-Newtonian Fluid Mech, 103, 251-260.

Phelps J H, Tucker C L (2009), 'An anisotropic rotary diffusion model for fibre orientation in shortand long-fibre thermoplastics', J Non-Newtonian Fluid Mech, 156, 165-176.

Pichelin E, Coupez T (1998), 'Finite element solution of the 3D mould filling problem for viscous incompressible Fluid', Comp Meth Appl Mech Eng, 163, 359-371.

Pichelin E, Coupez T (1999), 'A Taylor discontinuous Galerkin method for the thermal solution in 3d mould filling', Comp Meth Appl Mech Eng, 178, 153-169.

Prager S (1957), 'Stress-strain relation in a suspension of dumbbells', J Rheol, 1, 53-62.

Ramazani A, Ait-Kadi A and Grmela M (1997), 'Rheological modelling of short fiber thermoplastic composites' J. Non-Newtonian Fluid Mech., 73, 241-260.

Ramazani A, Ait-Kad A and M. Grmela M (2001) 'Rheology of fiber suspensions in viscoelastic media: experiments and model predictions' J. Rheol., 45, 945-962, 2001.

Ranganathan S, Advani S G (1991), 'On Modeling Fibre-Fibre Interactions of Flowing Suspensions in Homogeneous Flows', J Rheol, 35, 1499-1522.

Redjeb A (2007), 'Numerical simulation of fibre orientation in injection of reinforced thermoplastics', $\mathrm{PhD}$ thesis ENSMP, http://tel.archives-ouvertes.fr/tel-00332719/fr/

Redjeb A, Silva L, Laure P, Vincent M, Coupez T (2005), 'Numerical simulation of fibre orientation in injection moulding process', 21st Int. Polymer Processing Society Meeting, Leipzig, Germany.

Schiek R, Shaqfeh E (1997), 'Oscillatory shear of a confined fibre suspension', J Rheol, 41, 445-466.

Sepehr, M, Ausias, G, Carreau P J (2004), 'Rheological properties of short fibre filled polypropylene in transient shear flow', J Non-Newtonian Fluid Mechanics, 123(1), 19-32.

Shaqfeh E, Fredrickson G (1990), 'The hydrodynamic stress in a suspension of rods', Phys Fluids A, 2, 7-24.

Shen H B, Nutt S, Hull D (2004) 'Direct observation and measurement of fibre architecture in short fibre-polymer composite foam through micro-CT imaging', Comp Sci Technol, 64, 2113-2120.

Scheuer A, Abisset-Chavanne E, Chinesta F, Keunings R (2016), 'Second-gradient modelling of orientation development and rheology of dilute confined suspensions', J Non-Newtonian Fluid Mech., 237, 54-64.

Scheuer A, Abisset-Chavanne E, Chinesta F, Keunings R (2018), 'Microscopic modelling of orientation kinematics of non-spherical particles suspended in confined flows using unilateral mechanics', Comptes Rendus Mécanique, 346(1), 48-56. 
Silva L, Coupez T, Digonnet H, Laure P (2016), 'Multiphysics for Simulation of Forming Processes', In Heat Transfer in Polymer Composite Materials: Forming Processes, Boyard N (ed.), Wiley, 269300 .

Snook B, Guazzelli E, Butler J (2012), 'Vorticity alignment of rigid fibres in an oscillatory shear flow: role of confinement', Phys Fluids 24, 121702.

Souloumiac B, Vincent M (1998), 'Steady shear viscosity of short fibre suspensions in thermoplastics', Rheol. Acta 37 (3), 289-298.

Sommer D E, Favaloro A J and R. Byron Pipes R B (2018), 'Coupling anisotropic viscosity and fiber orientation in applications to squeeze Flow', J. Rheol. 62(3), 669-679.

Stover C A, Cohen C (1990), 'The motion of rod-like particles in pressure-driven flow between two flate plates', Rheol Acta 29, 192-203.

Stover C A, Koch DL, Cohen C (1992), 'Observations of fibre orientation in simple shear-flow of semidilute suspensions', J Fluid Mech, 238, 277-296.

Suuronen J P, A. Kallonen A, M. Eik M, J. Puttonen J, R. Serima R, H. Herrmann H (2013), Analysis of short fibres orientation in steel fibre-reinforced concrete (SFRC) by x-ray tomograph, J Mater Sci, 48 1358-1367.

Taylor G (1923), 'The motion of ellipsoidal particles in a viscous fluid', Proc. R. Soc. London A,103, 58-61.

Toll S (1993), 'Note: On the Tube Model for Fibre Suspensions', J Rheology, 37, 123-125.

Toll S (1998), 'Packing Mechanics of Fibre Reinforcements', Polym Eng Sci, 38, 1337-1350.

Tremblay S R, Lafleur P G, Ait-Kadi A (2000), 'Effects of Injection Parameters on Fibre Attrition and Mechanical Properties of Polystyrene Molded Parts', J. Inj. Molding Technol, 4(1), 1-7.

Trevelyan B J, Mason S G (1951), 'Particle motions in sheared suspensions I. Rotations', J Colloid Sci, 6, 354-367.

Tucker C L (1991), 'Flow regimes for fibre suspensions in narrow gap', J Non-Newtonian Fluid Mech, $39,239-268$.

Tucker C L, Advani S G (1994), 'Processing of Short-Fibre Systems, Flow and Rheology in Polymer Composites Manufacturing', Elsevier, Amsterdam.

Tseng H-C, Chang R-Y, Hsu C-H (2013), 'Phenomenological improvements to predictive models of fibre orientation in concentrated suspensions', J Rheol, 57, 1597-631.

Tseng H-C, Chang R-Y, Hsu C-H (2016), 'An objective tensor to predict anisotropic fibre orientation in concentrated suspensions', $J$ Rheol, 60, 215-24.

Tseng H-C, Chang R-Y, Hsu C-H (2017), 'Numerical predictions of fibre orientation and mechanical properties for injection-molded long-glass-fibre thermoplastic composites', Composites Science and Technology, 150, 181-186. 
Tseng H-C, Chang R-Y, Hsu C-H (2018), 'The use of principal spatial tensor to predict anisotropic fibre orientation in concentrated fibre suspensions', J Rheol, 62, 313-20.

Tseng H-C and Favaloro A J (2019), 'The use of informed isotropic constitutive equation to simulate anisotropic rheological behaviors in fiber suspensions', J Rheology 63, 263.

Verleye V (1995), 'Simulation Numérique de l'orientation de fibres en moulage par injection de pieces complexes en matériaux composites', PhD thesis UCL

Ville L, Silva L, Coupez T (2011), 'Convected level set method for the numerical simulation of fluid buckling', Int J Numer Meth Fluids, 66: 324-344.

Vincent M, Giroud T, Clarke A, Eberhardt C (2005), 'Description and modeling of fibre orientation in injection molding of fibre reinforced thermoplastics', Polymer, 46(17), 6719-6725.

Vincent M (2009), 'Flow induced fibre micro-structure in injection molding of fibre reinforced materials', in Injection molding, technology and fundamentals, Kamal M R, Isayev A I, Liu S J (eds), Carl Hanser Verlag, Munich, 253-272.

Viguié J, Latil P, Orgéas L, Dumont P J J, Rolland du Roscoat S, Bloch J F, Marulier C, O. Guiraud O (2013), 'Finding fibres and their contacts within 3D images of disordered fibrous media', Compos Sci Technol, 89, 202-210.

Wang J, O'Gara J F, Tucker C L (2008), 'An objective model for slow orientation kinetics in concentrated fibre suspensions: Theory and rheological evidence', J Rheol, 52, 1179-1200.

Wegner S, Borzsonyi T, Bien T, Rose G, Stannarius R (2012), 'Alignment and dynamics of elongated cylinders under shear', Soft Matter, 8, 10950-10958.

Wetzel E D, Tucker C L (1999), 'Area Tensors for modeling microstructure during laminar liquidliquid mixing', Int J Multiphase Flow, 25, 35-61.

Wittemann F, Maertens R, Kärger L, Henning F (2019), 'Injection molding simulation of short fiber reinforced thermosets with anisotropic and non-Newtonian flow behavior', Composites Part A 124, 105476.

Yamane Y, Kaneda Y, Dio M (1994), 'Numerical simulation of semi-dilute suspensions of rodlike particles in shear flow', J Non-Newtonian Fluid Mech, 54, 405-421.

Yasuda K, Mori N, Nakamura K (2002), 'A new visualization technique for short fibres in a slit flow of fibre suspensions’, Int J Eng S. C, 40 1037-1052. 


\begin{tabular}{|c|c|c|}
\hline Position & $\mathrm{Q}=9 \mathrm{~cm}^{3} \cdot \mathrm{s}^{-1}$ & $\mathrm{Q}=122 \mathrm{~cm}^{3} \cdot \mathrm{s}^{-1}$ \\
\hline First rib & 1200 & 1300 \\
\hline Plaque near the first rib & 1200 & 900 \\
\hline Plaque between $4^{\text {th }}$ and $5^{\text {th }}$ rib & 1000 & 1000 \\
\hline Plaque near the $5^{\text {th }}$ rib & 900 & 1000 \\
\hline $5^{\text {th }}$ rib & 1000 & \\
\hline
\end{tabular}

Table 20.1: Average length in $\mu m$ for different positions in the part shown in figure 20.2. 


\begin{tabular}{|c|c|c|c|}
\hline & PAA50 & P30YM240 & P40YM243 \\
\hline$\tau_{c}$ & $0.154 \mathrm{MPa}$ & $0.0411 \mathrm{MPa}$ & $0.02626 \mathrm{MPa}$ \\
\hline$\eta_{0}\left(\mathrm{~T}_{\mathrm{ref}}\right)$ & 570 Pa.s & 252 Pa.s & 636.6 Pa.s \\
\hline$\alpha$ & 0.55 & 1 & 1 \\
\hline$m$ & 0.3 & 0.22 & 0.271 \\
\hline $\mathrm{T}_{\mathrm{ref}}$ & $549 \mathrm{~K}$ & $523 \mathrm{~K}$ & $493 K$ \\
\hline$\beta_{T}$ & 7764 mole.K & 4450 mole.K & 4450 mole. $\mathrm{K}$ \\
\hline$k_{T}$ & $0.3 \mathrm{~W} / \mathrm{m} . \mathrm{K}$ & $0.25 \mathrm{~W} / \mathrm{m} . \mathrm{K}$ & $0.15 \mathrm{~W} / \mathrm{m} . \mathrm{K}$ \\
\hline$C_{p}$ & $1766 \mathrm{~J} / \mathrm{Kg} . \mathrm{K}$ & $2180 \mathrm{~J} / \mathrm{Kg} . \mathrm{K}$ & $3100 \mathrm{~J} / \mathrm{Kg} . \mathrm{K}$ \\
\hline$\rho$ & $1522 \mathrm{Kg} / \mathrm{m}^{3}$ & $1000 \mathrm{Kg} / \mathrm{m}^{3}$ & $1000 \mathrm{Kg} / \mathrm{m}^{3}$ \\
\hline
\end{tabular}

Table 20.2: Parameters used in equations [20.52] and [20.353] for PAA50, Stamax P30YM240 and P40YM243: $C_{p}$ is the specific heat, $\kappa$ is the thermal conductivity. 
Figure 20.1: Fibre length repartition in a $30 \mathrm{wt} \%$ long and short glass fibre reinforced polypropylene moulded plaque

Figure 20.2: Geometry of the plaque with 7 ribs: the size is $150 \times 150 \times 3 \mathrm{~mm}$ and the ribs spaced of $25 \mathrm{~mm}$

Figure 20.3: Fibre weight concentration (wt $\%$ ) in the part shown on figure 2

Figure 20.4: Pictures of fibre reinforced polymer moulded plaque (polypropylene having $30 \%$ of 12 $\mathrm{mm}$ long fibers). The thickness is in the vertical direction. On the left (a) the flow direction is perpendicular to the observation plane. On the right (b) the flow direction is parallel to the large side of the picture.

Figure 20.5: Upper graph: compression of a concentrated suspension of quasi-ordered fibers with 3D in situ observations of its deformation (Latil et al., 2011). Evolution of the compression stress $\sigma$ as a function of the fiber content $\phi$ and corresponding evolution of the fibrous microstructure. Lower graph: corresponding evolution of the average number of contacts $\bar{z}$ as a function of the fiber content $\phi:$ the marks represent the experimental data, the continuous line represents the prediction given by Toll's model (equation 20.2) and the dotted line represents the prediction of the tube model without the spatial homogeneity assumption.

Figure 20.6: Orientation tensor component in the flow direction for four plaque thicknesses from 1.1 to $5 \mathrm{~mm}$ (after Vincent, 2009)

Figure 20.7: Ellipsoid representation of orientation tensor $\boldsymbol{a}_{2}$.

Figure 20.8: Mould schematic view (a) and zoom (b) on the inlet with positions of sensors. 
Figure 20.9: Isovalues of $a_{11}$ at the end of filling in a cross section $\left(i_{1}, i_{2}, i_{3}=0\right)$ for the PAA50 with isotropic initial orientation, $N_{p}=0$ and $C_{I}=0.001$

Figure 20.10: Values of orientation tensor coefficient $a_{11}$ for the four sensors A (a), B (b), C (c), D (d) for PAA50 and isotropic initial orientation: $C_{I}=0.001,0.04 ; N_{p}=0,100$.

Figure 20.11: Values of orientation tensor coefficient $a_{11}$ in the four areas A (a), B (b), C (c), D (d) for PAA50 and unidirectional initial orientation: $C_{I}=0.001,0.04 ; N_{p}=0,100$.

Figure 20.12: Geometry of a cross with thin walls (a); focus on the weld line located on the wall (b)

Figure 20.13: Isovalues of $a_{11}, a_{22}$ on the wall where is located the weld line

Figure 20.14: Ellipsoids represented orientation tensor coloured by Von Mises scalar for the plaque with ribs: isotropic orientation (white), unidirectional orientation (black)

Figure 20.15: Final arch drawn orientation near the third rib (a); comparison with the experiment (b) 

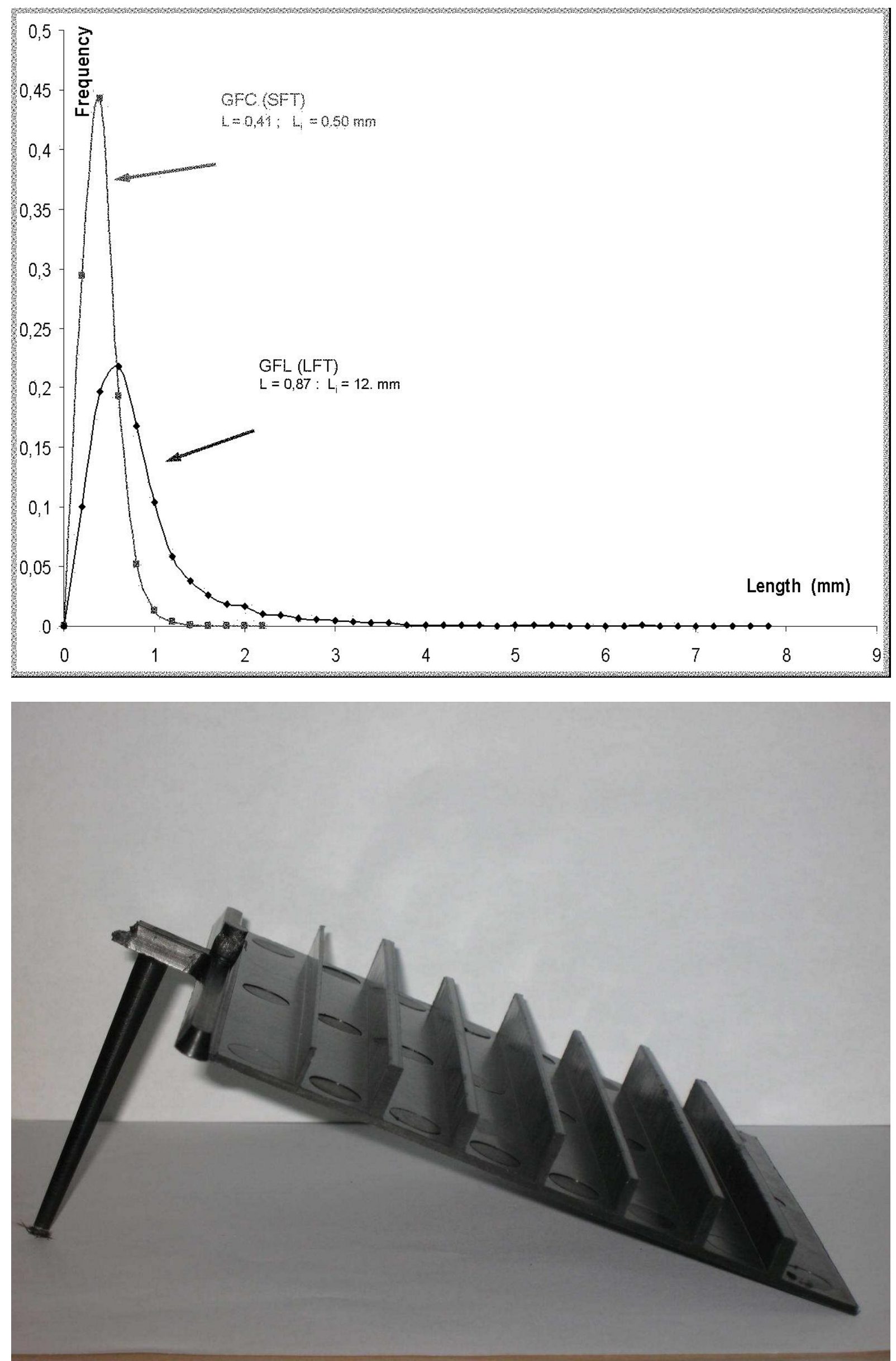

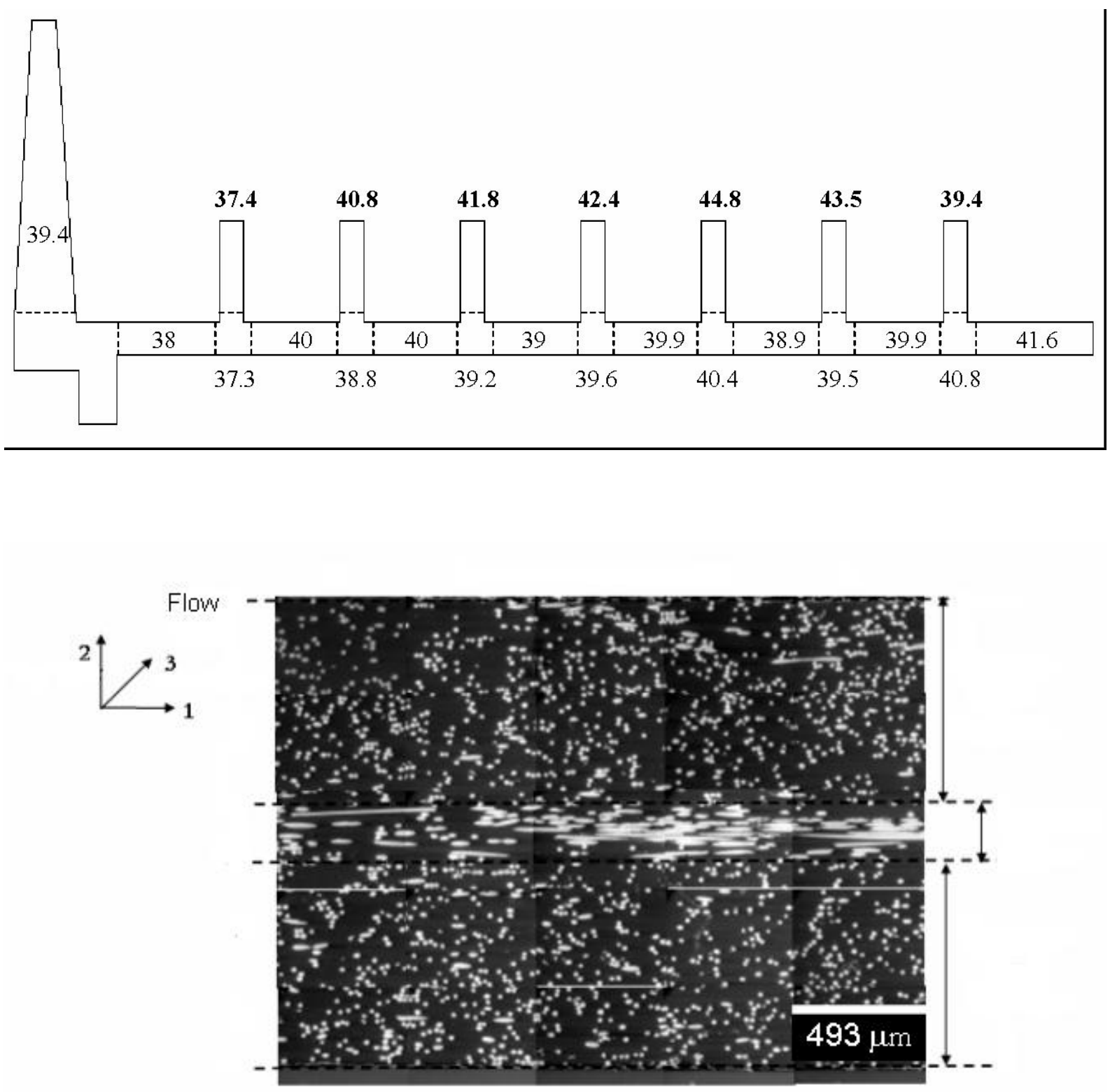


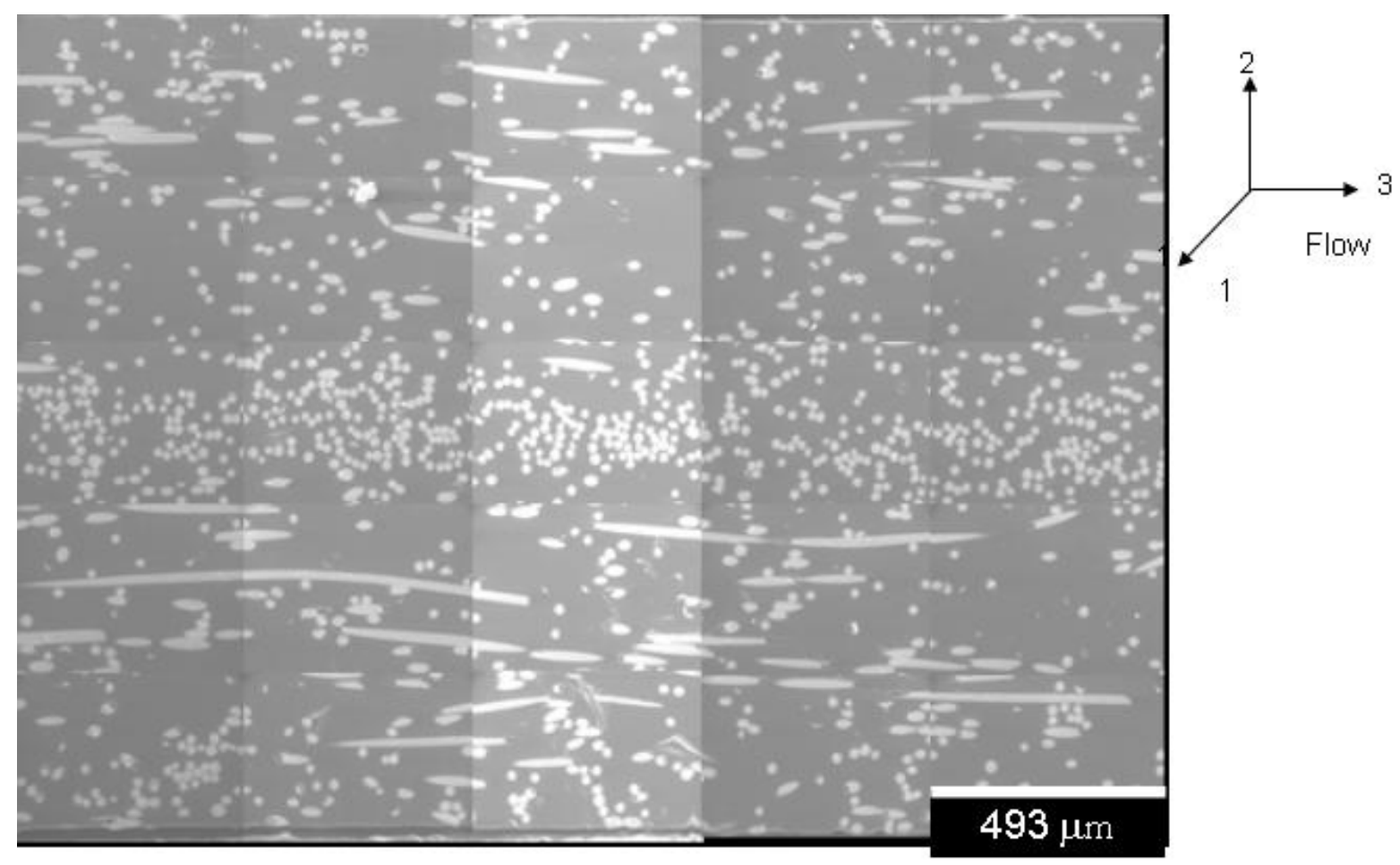



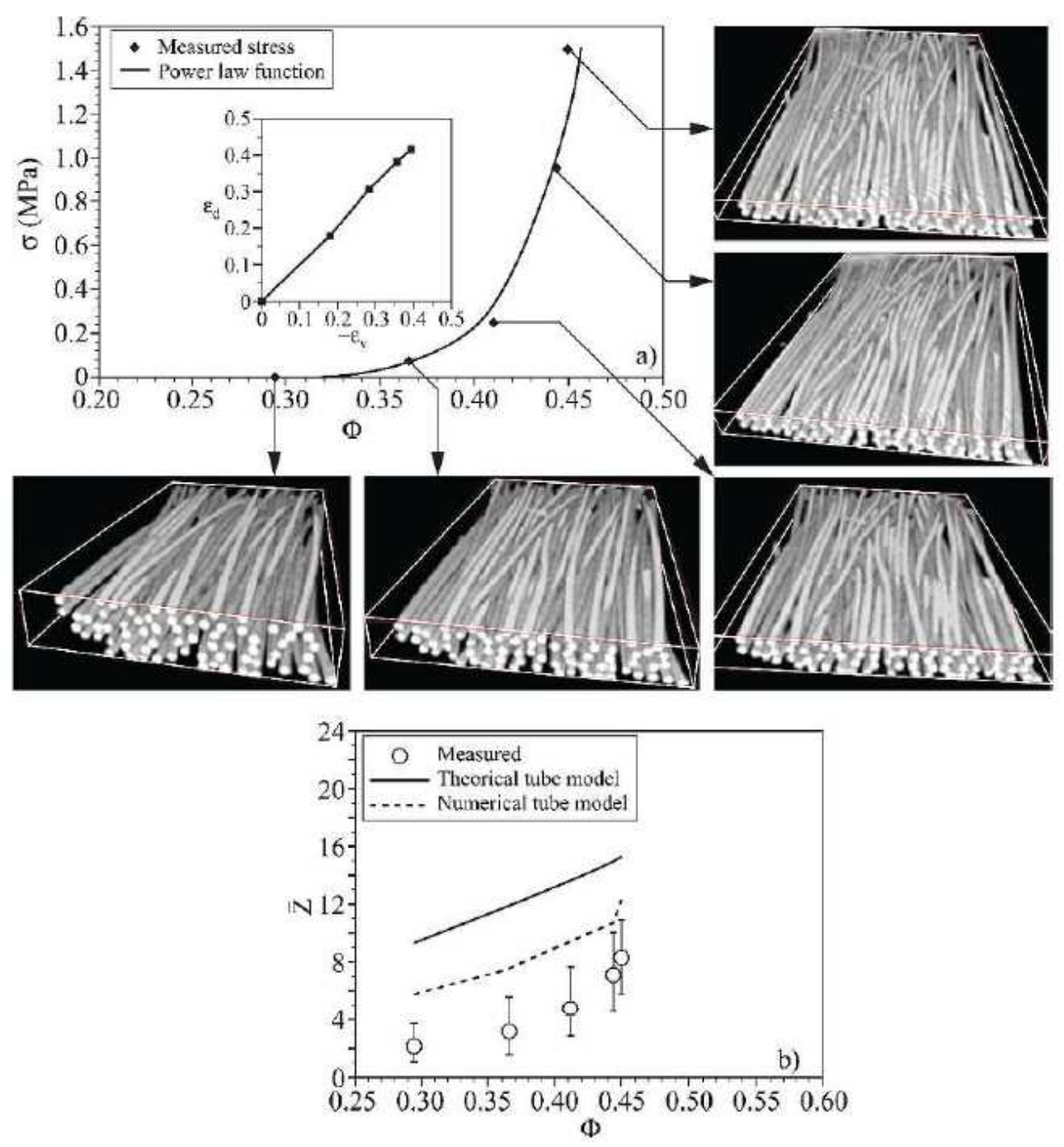

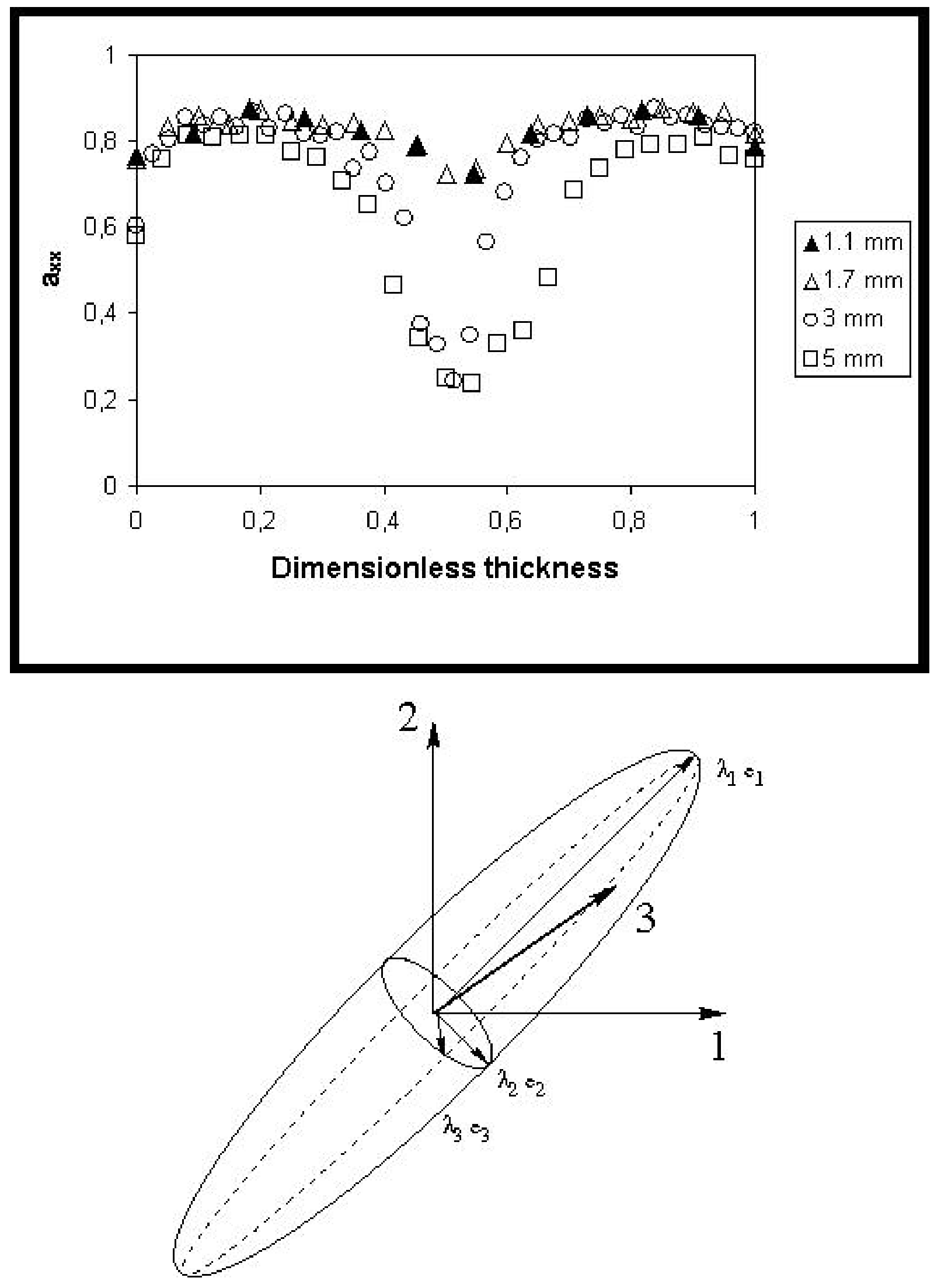


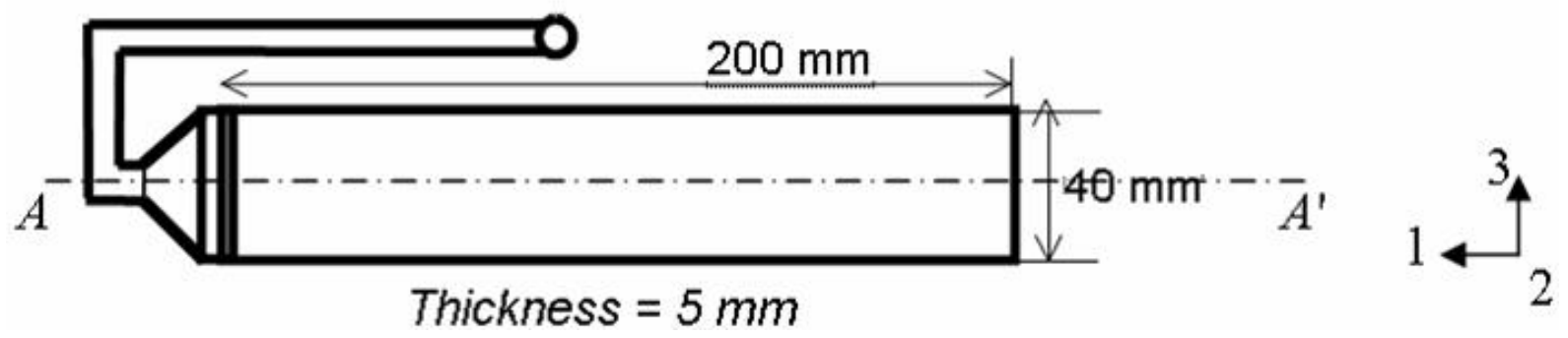

cut plane $\mathrm{x}_{2}=0$ :
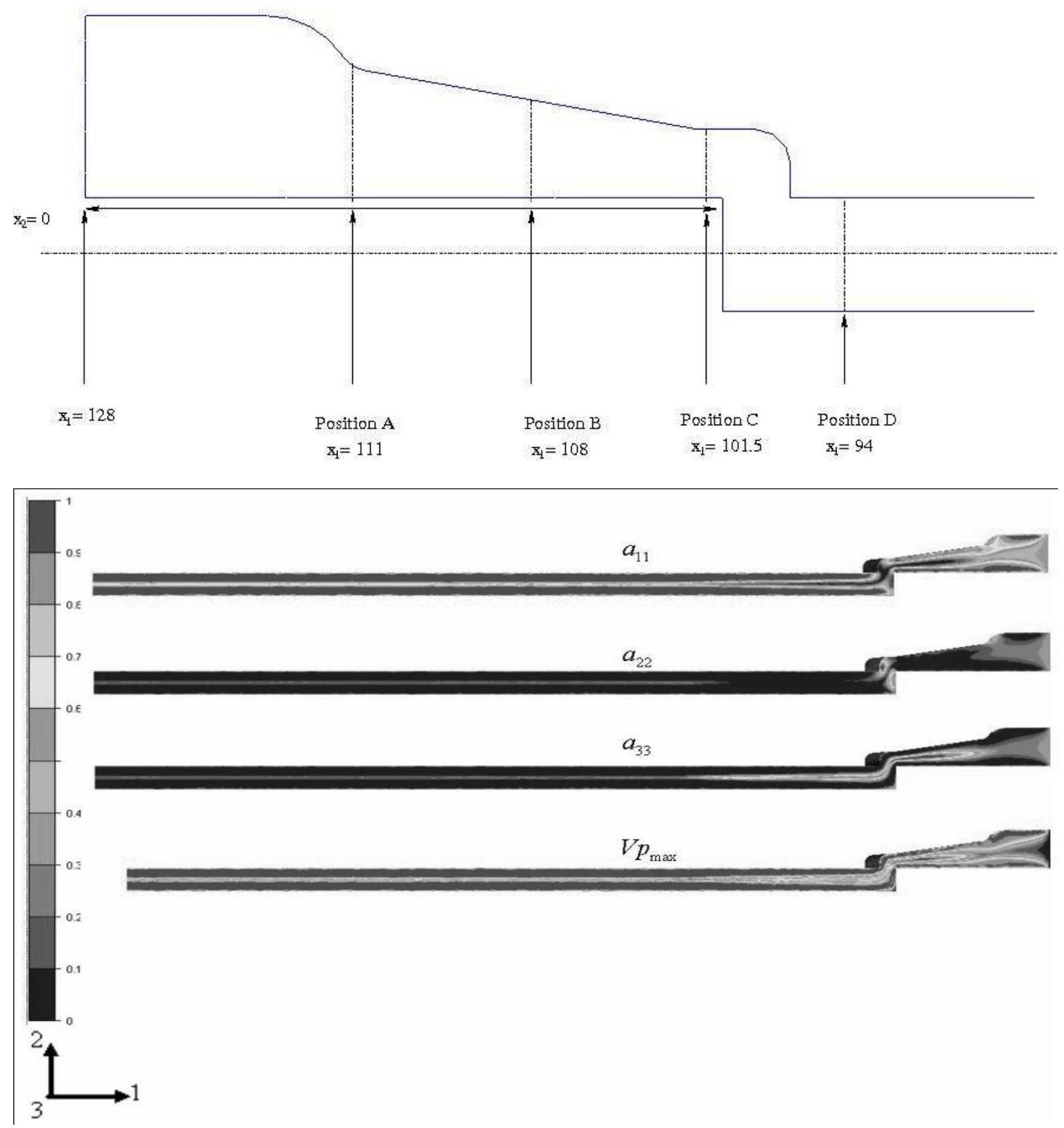


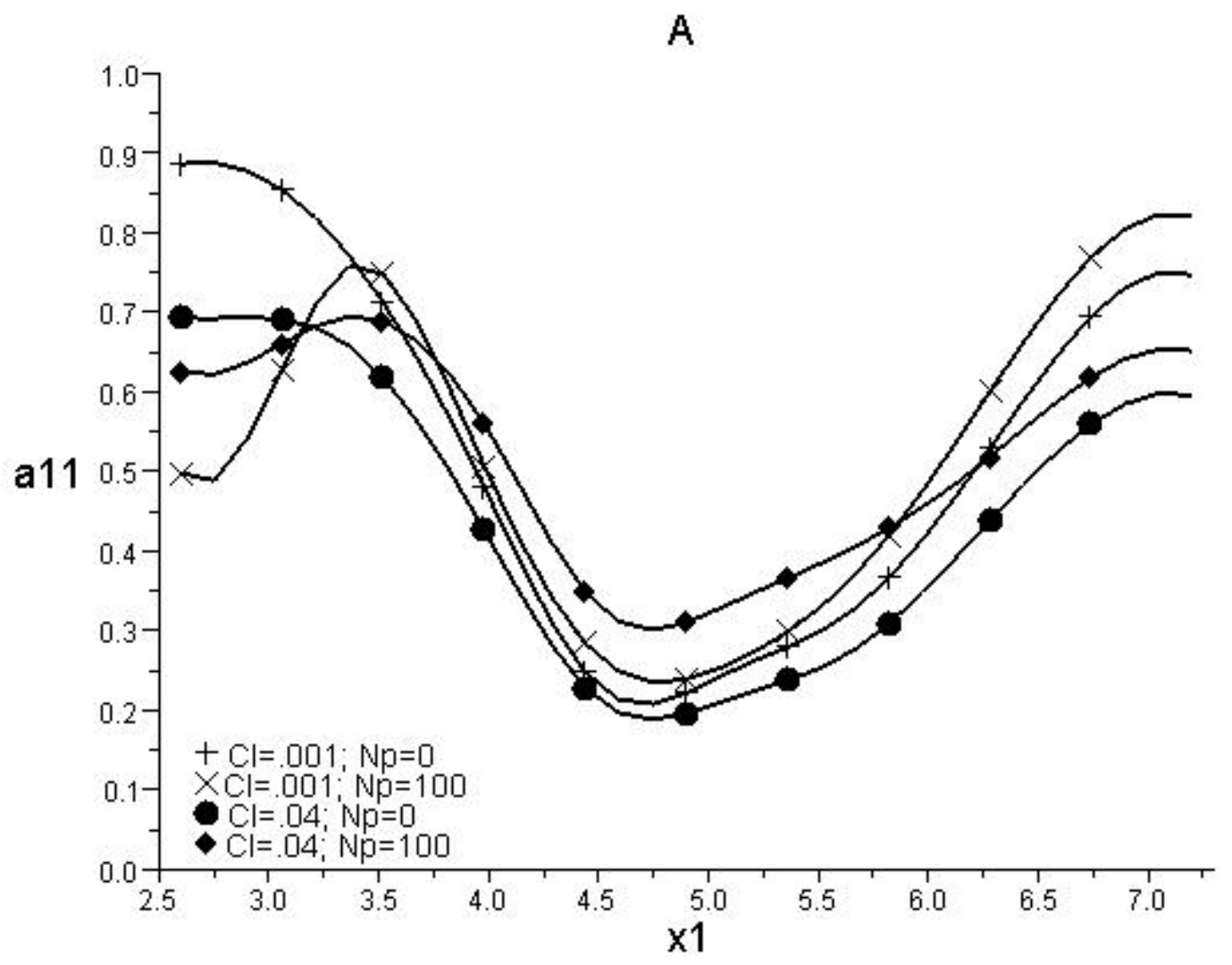

B

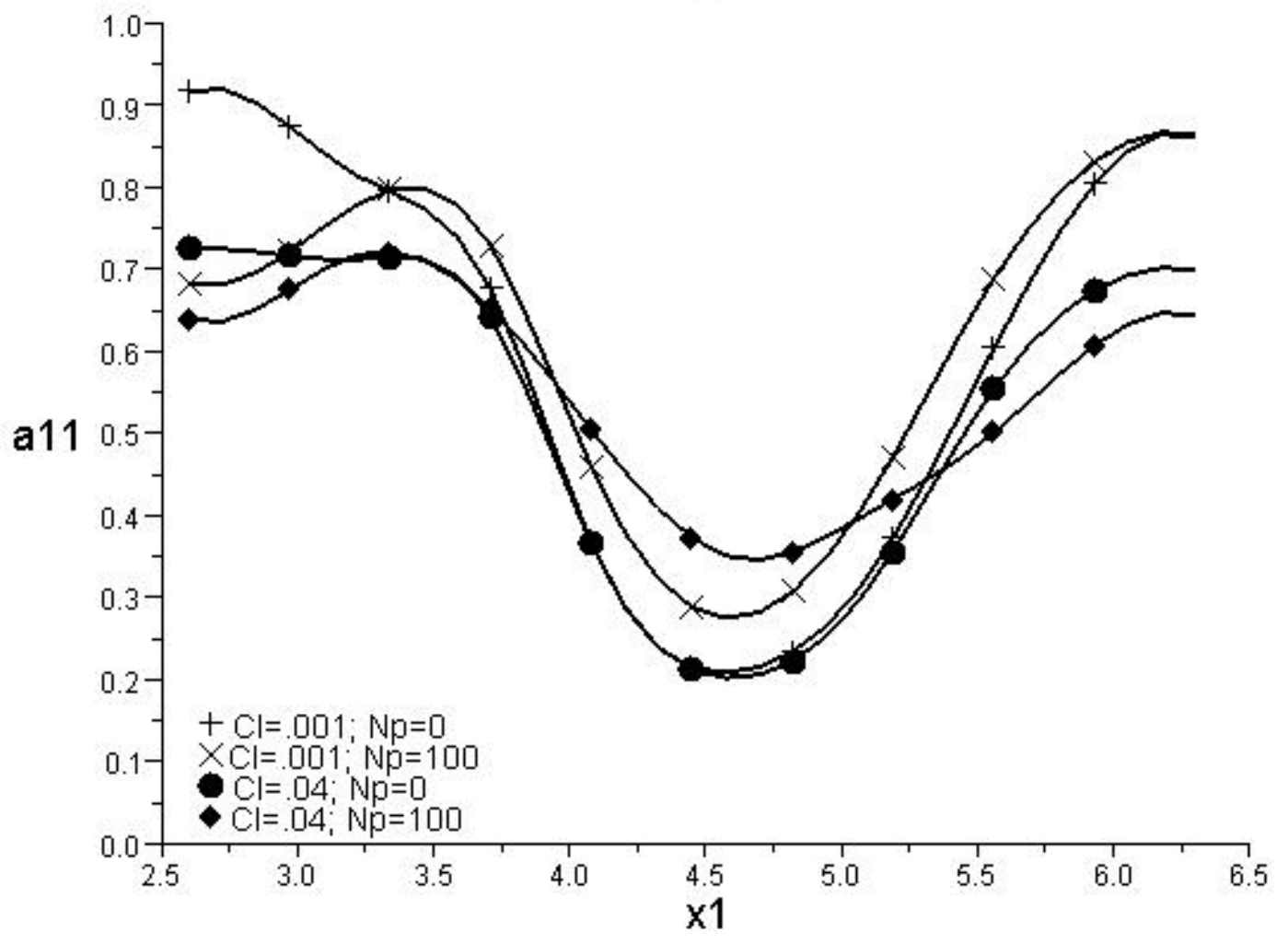


C

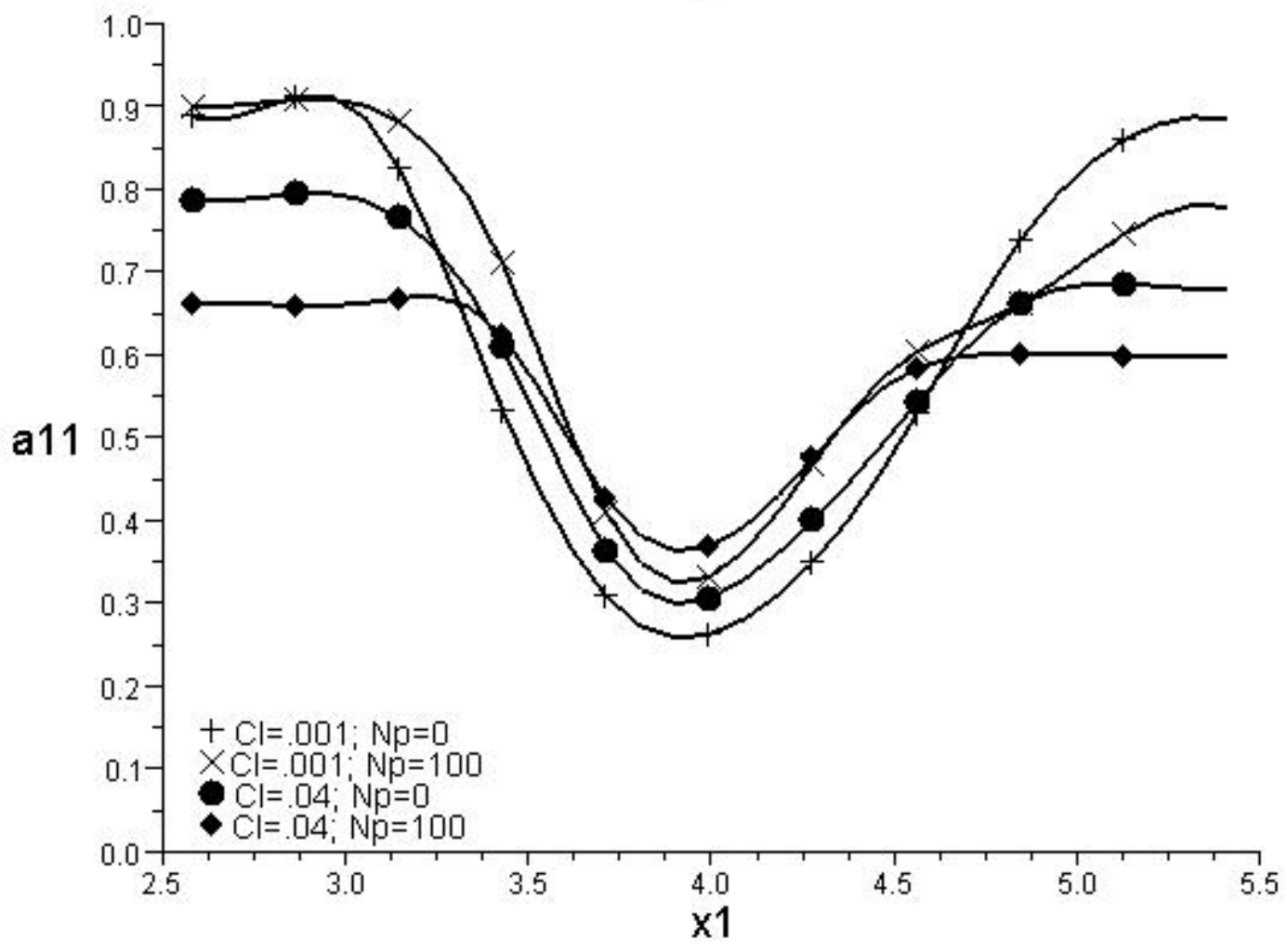

D

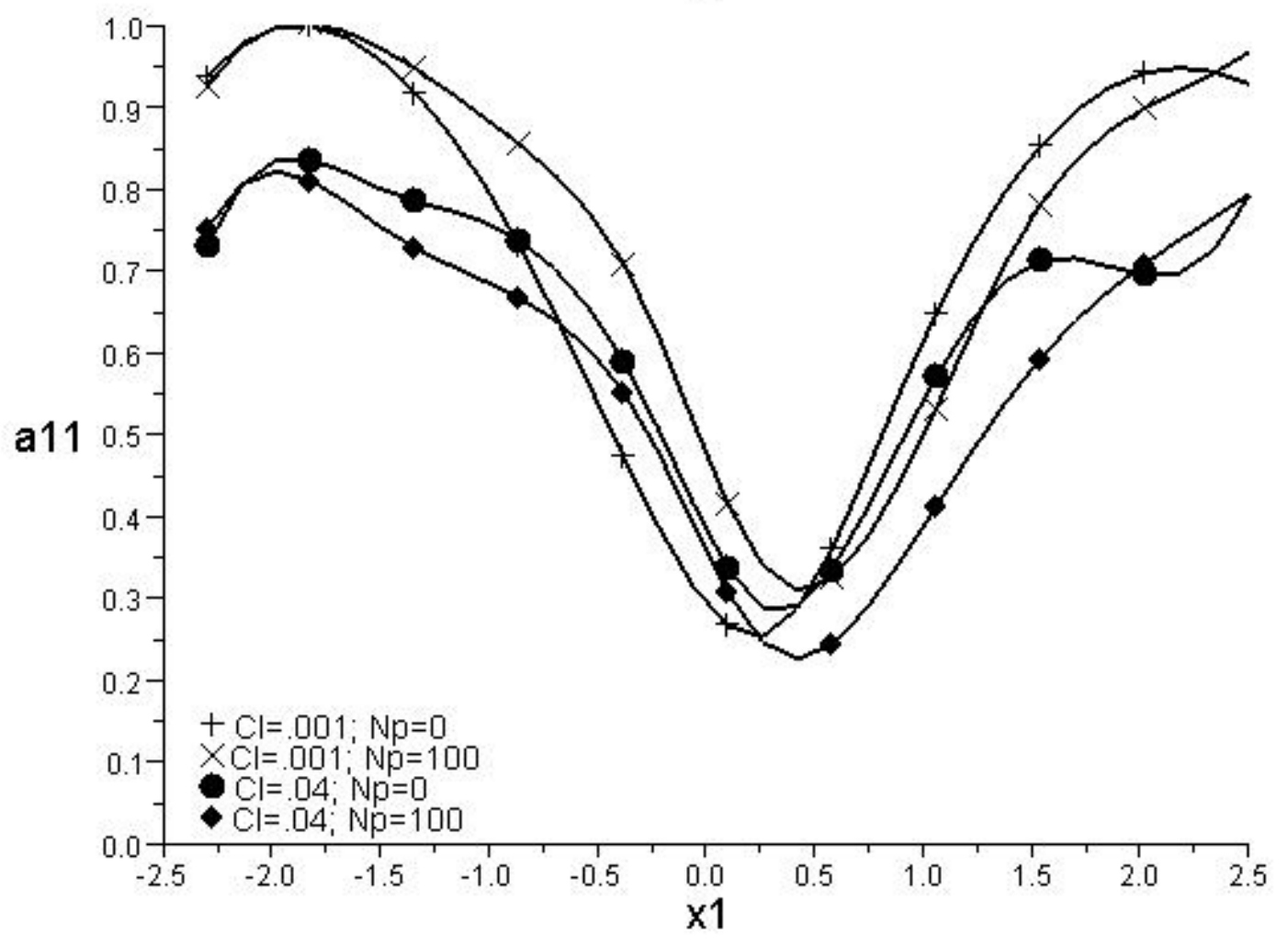




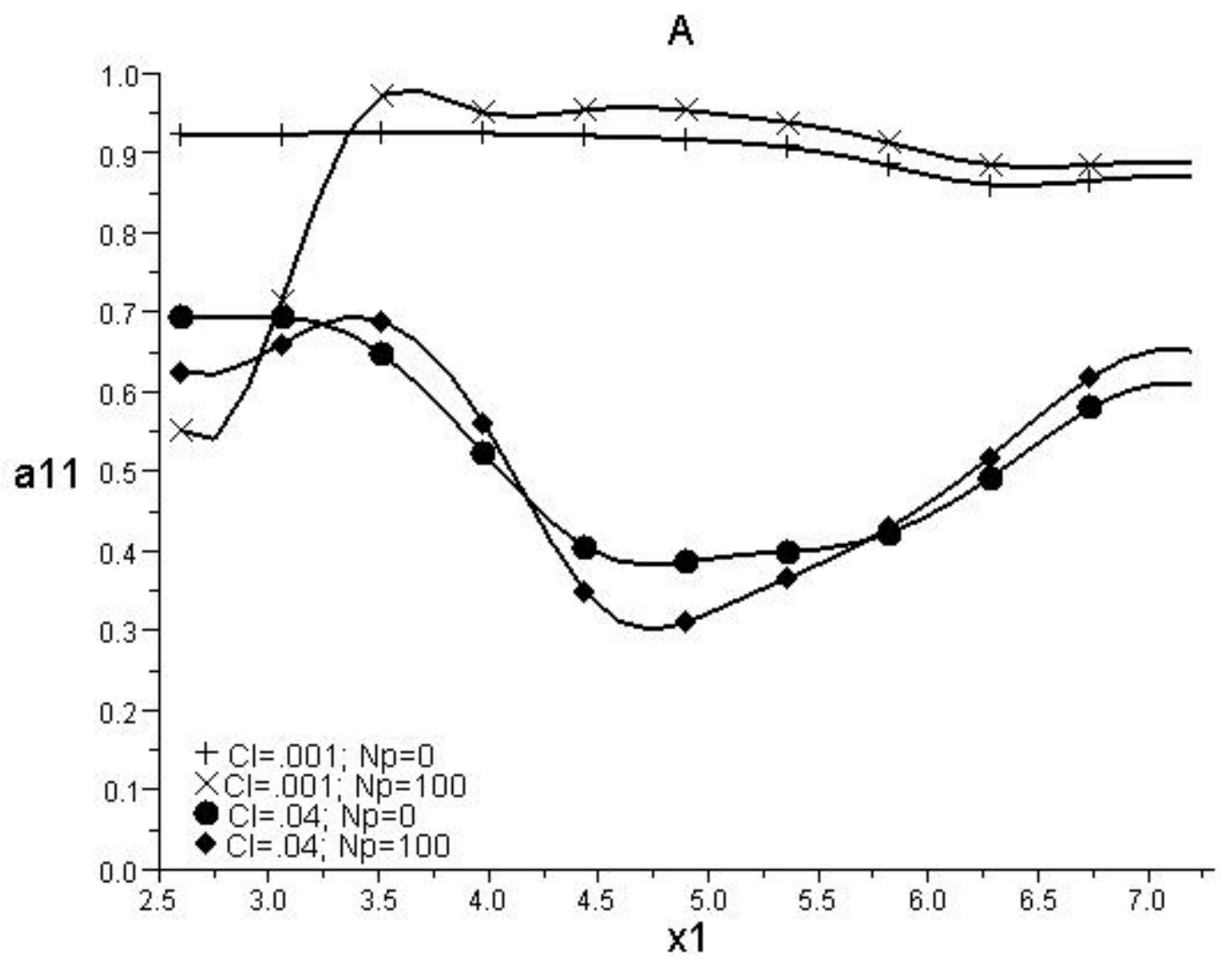

B

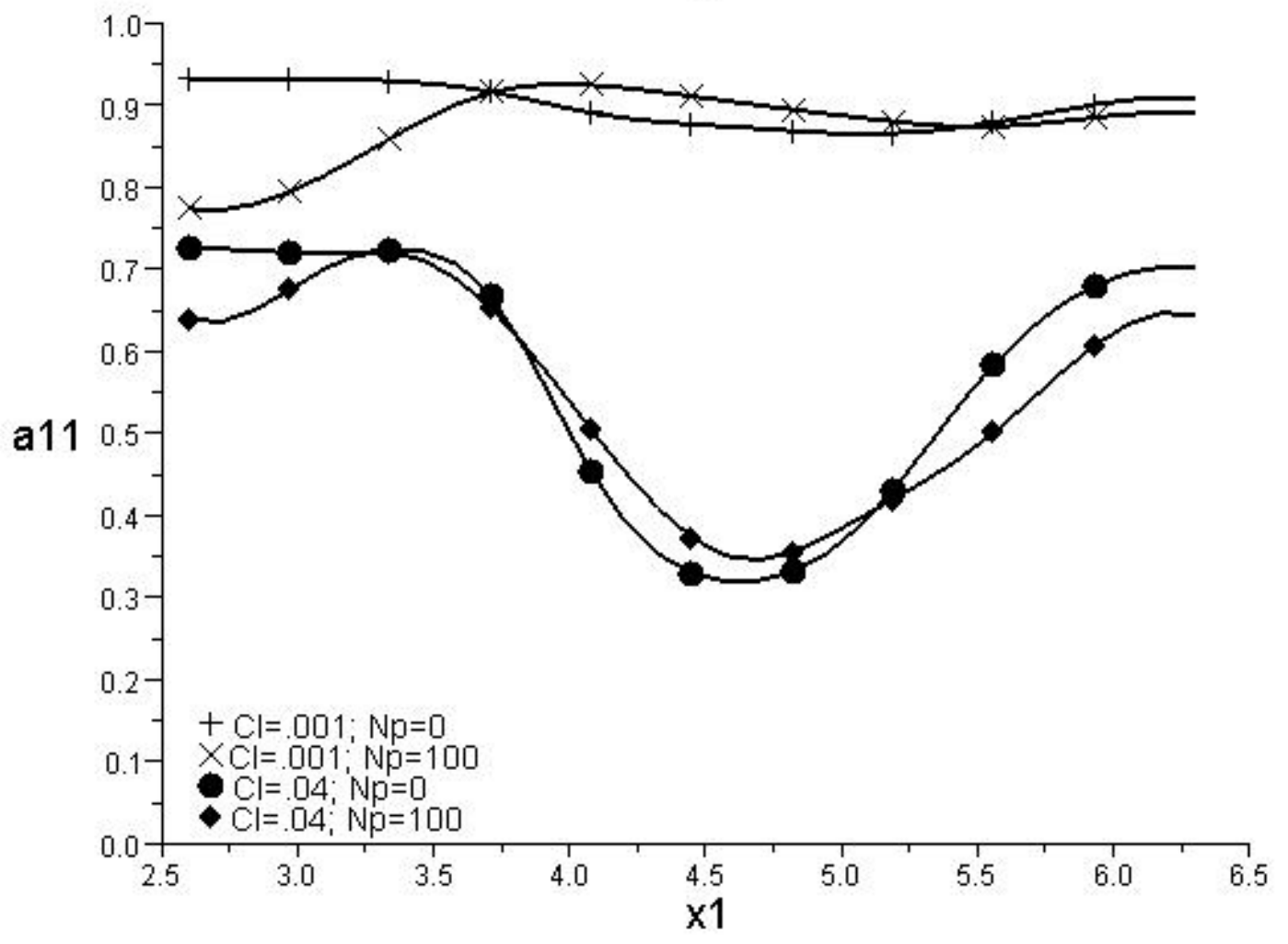


C

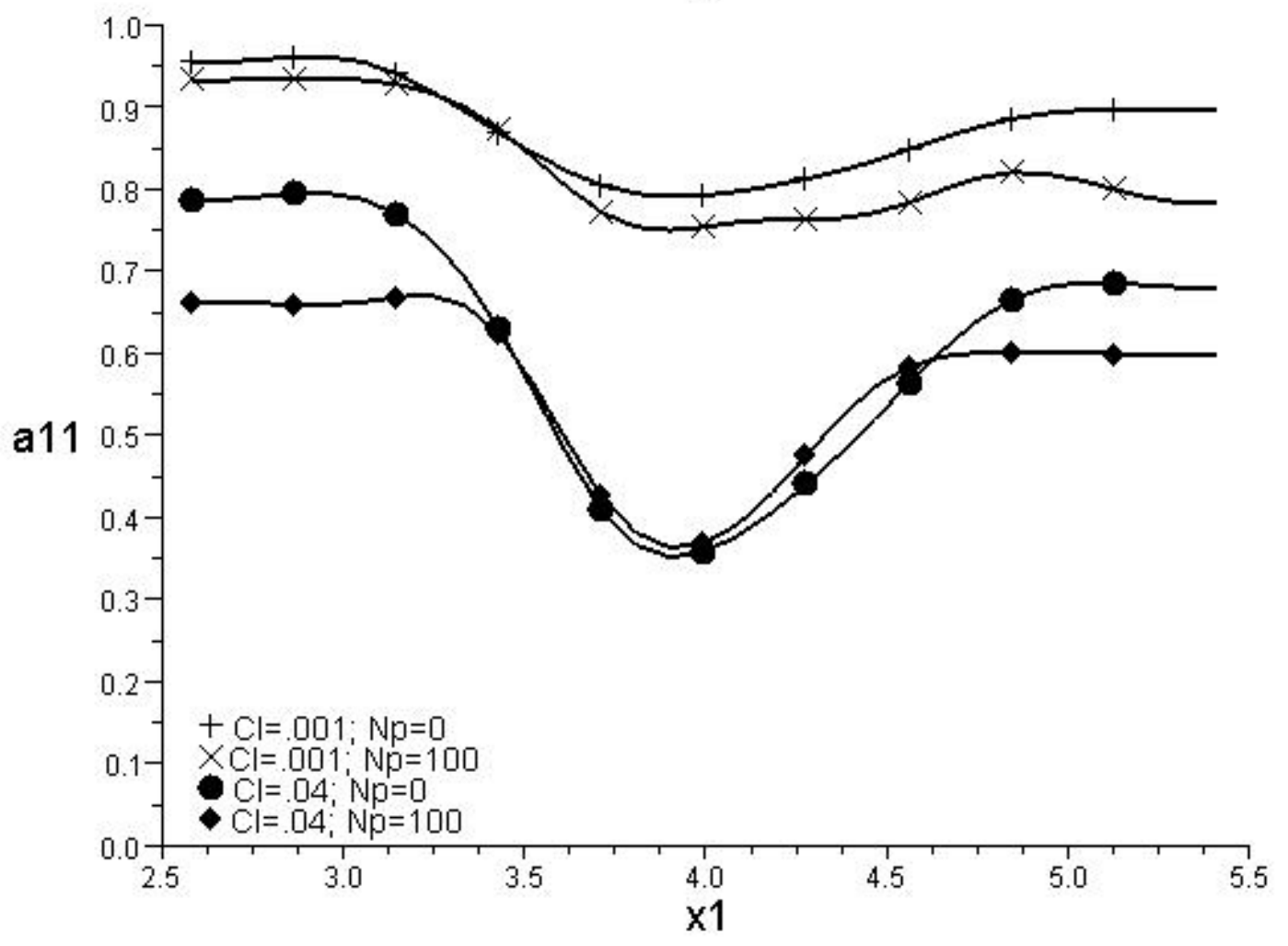

D

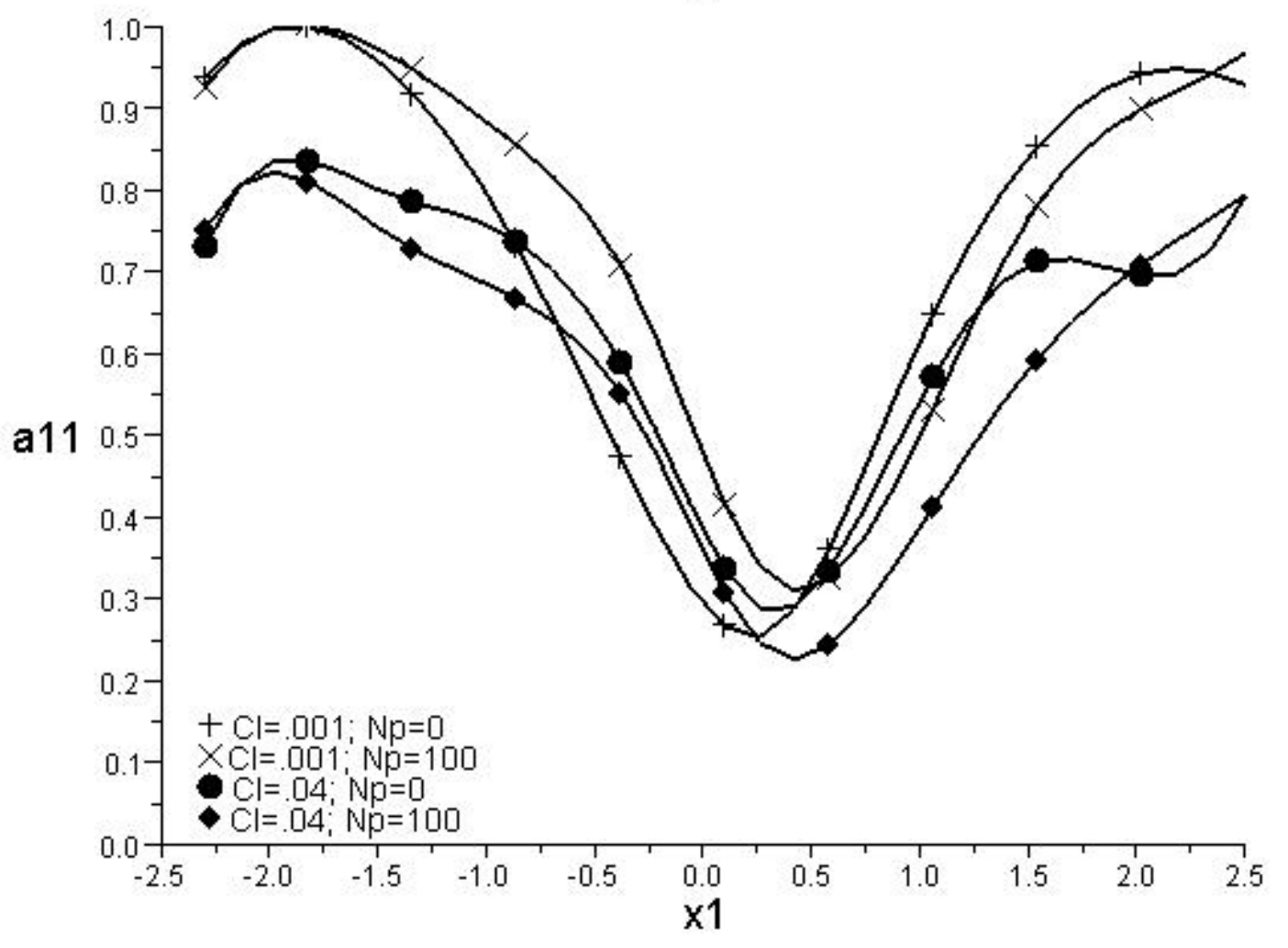




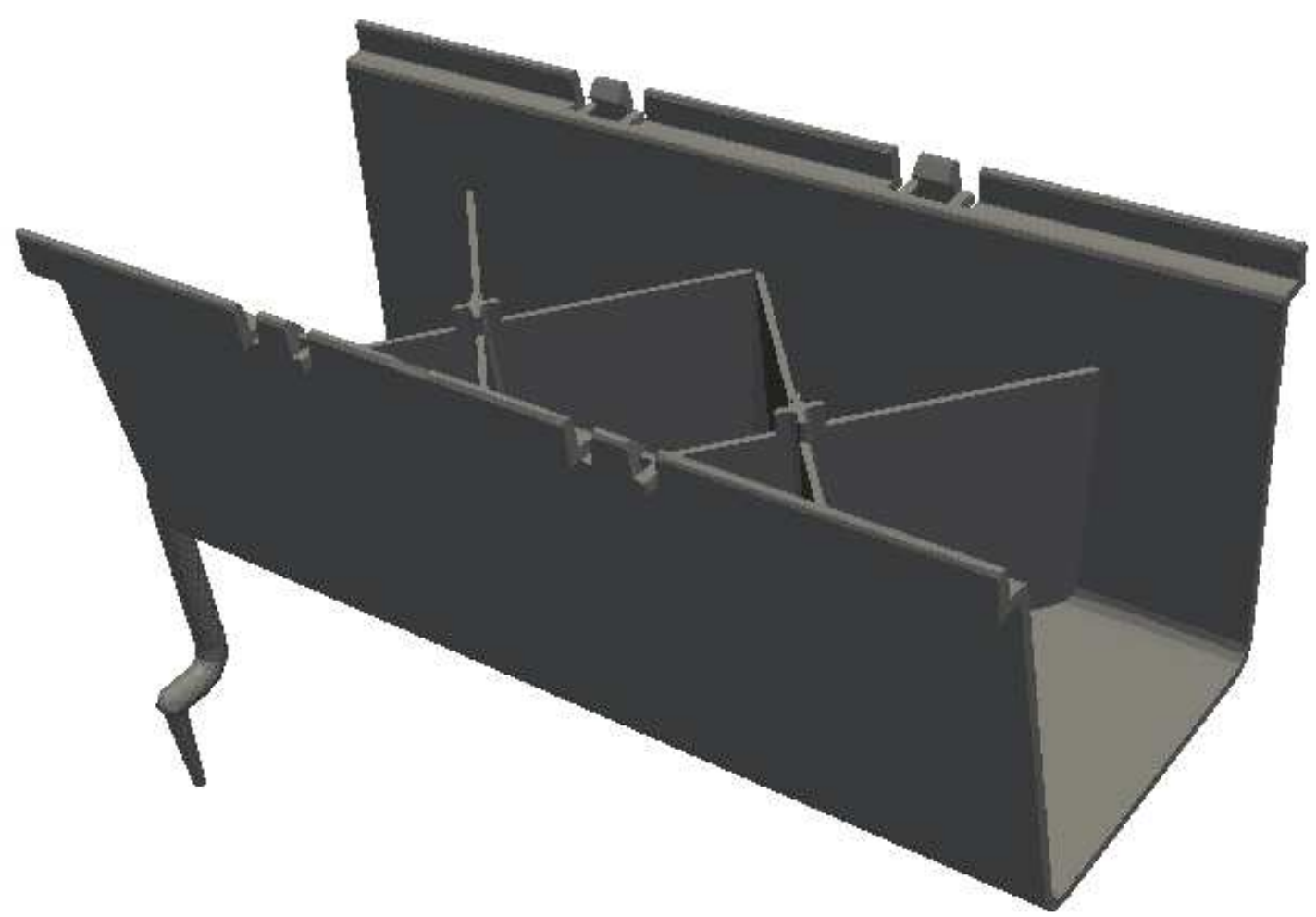




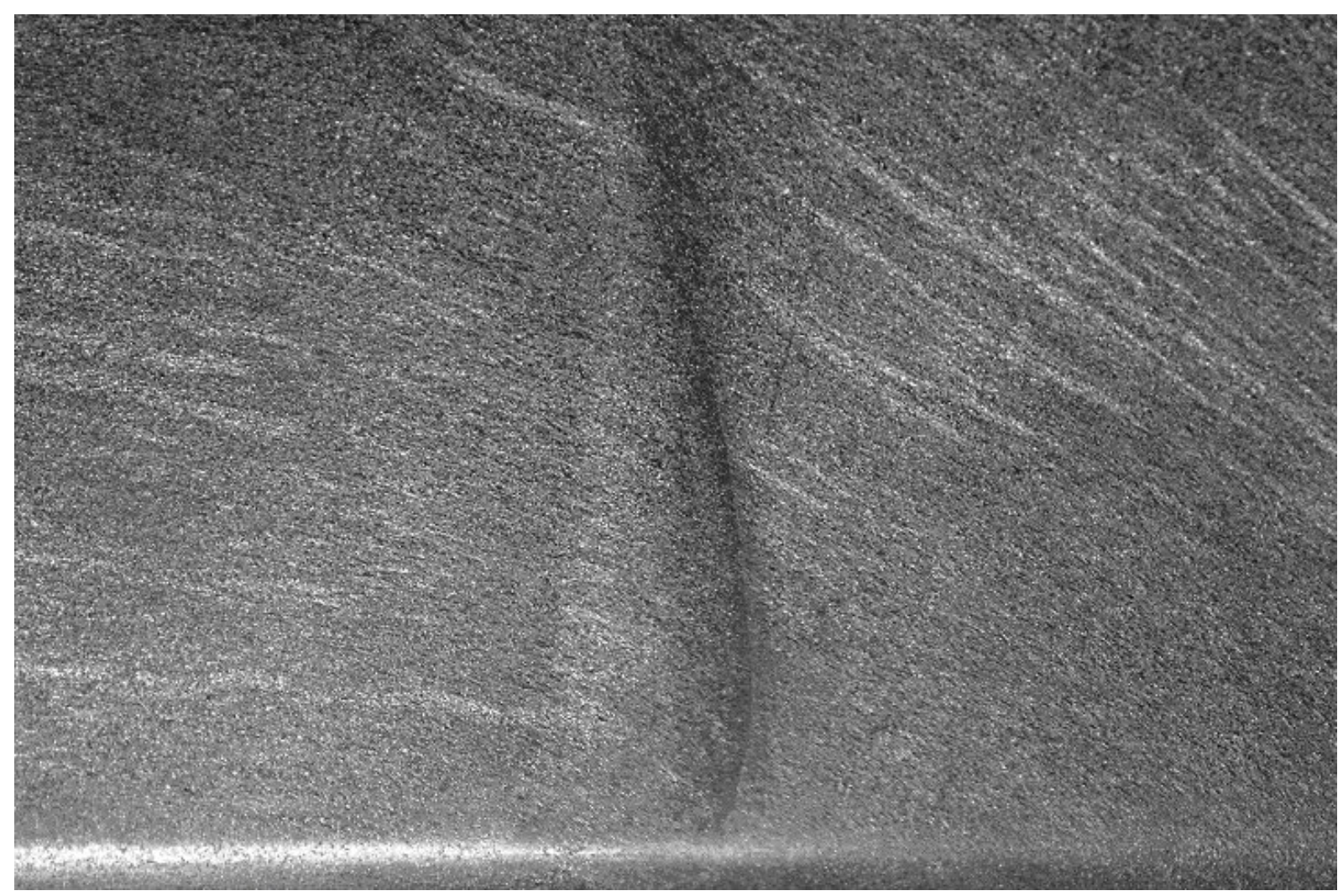



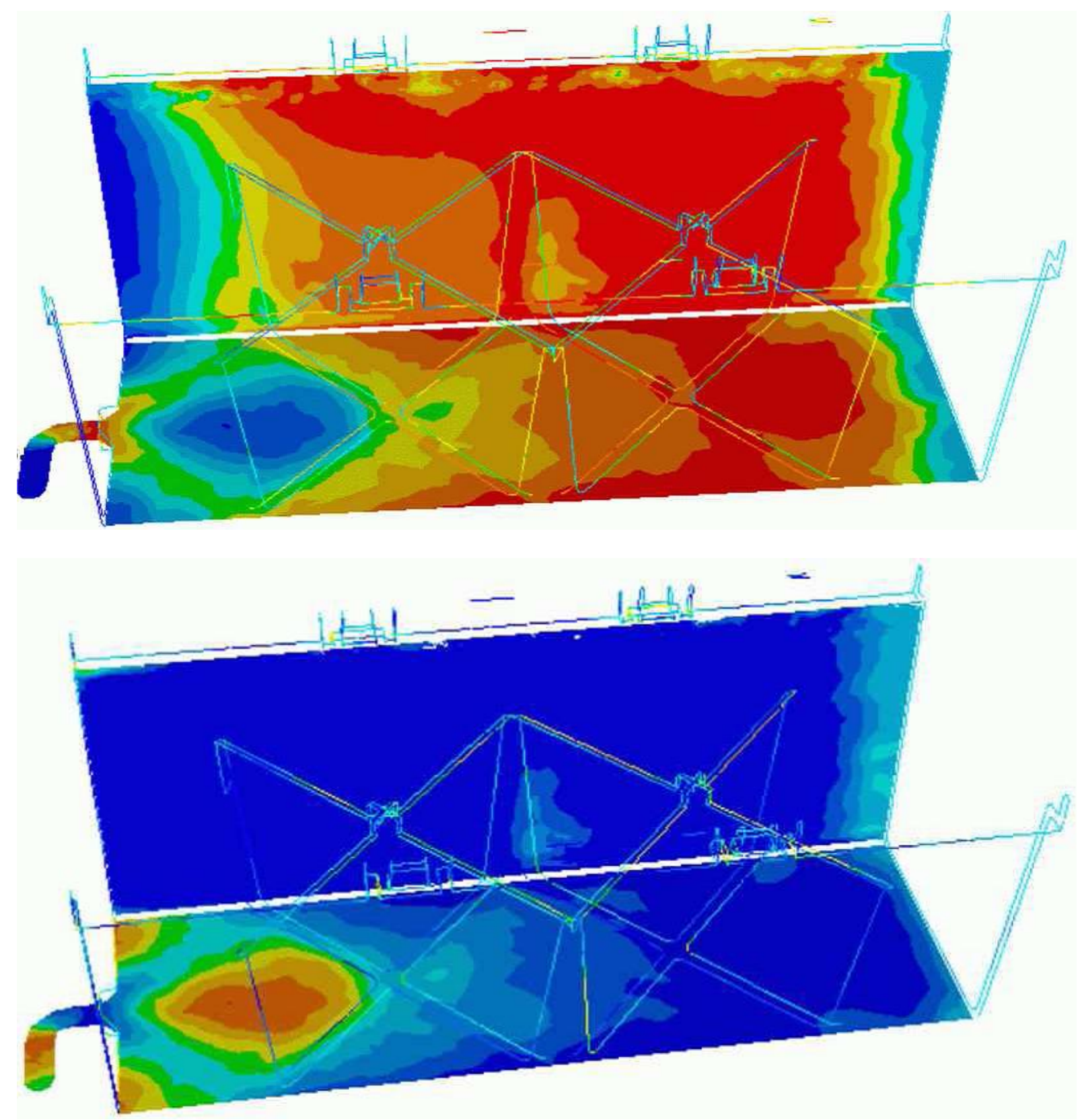

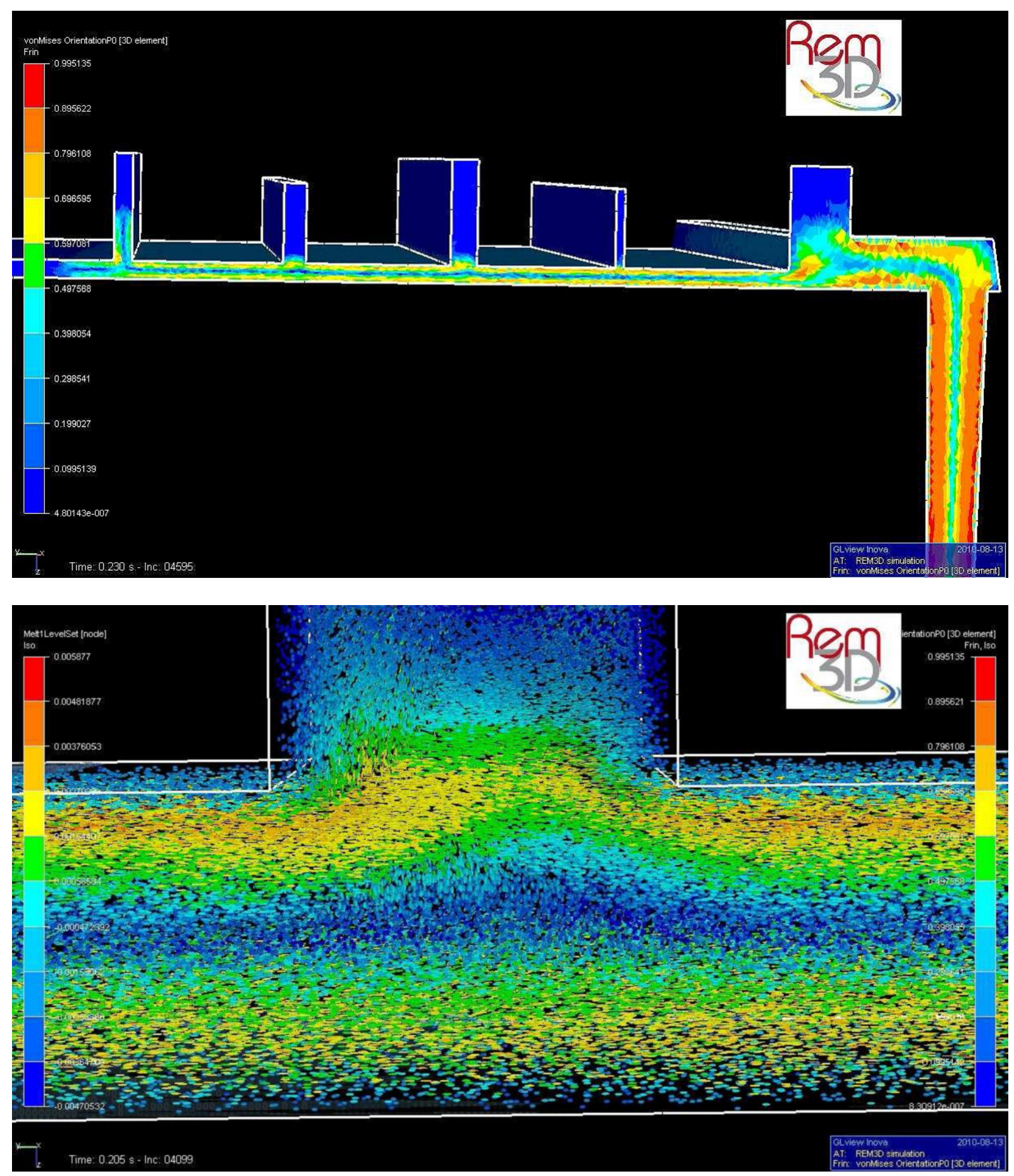